\title{
STABILITY OF $\mathbb{Z}^{2}$ CONFIGURATIONS IN 3D
}

\author{
LAURENT BÉTERMIN, MANUEL FRIEDRICH, AND ULISSE STEFANELLI
}

\begin{abstract}
Inspired by the issue of stability of molecular structures, we investigate the strict minimality of point sets with respect to configurational energies featuring two- and three-body contributions. Our main focus is on characterizing those configurations which cannot be deformed without changing distances between first neighbors or angles formed by pairs of first neighbors. Such configurations are called angle-rigid.

We tackle this question in the class of finite configurations in $\mathbb{Z}^{2}$, seen as planar three-dimensional point sets. A sufficient condition preventing angle-rigidity is presented. This condition is also proved to be necessary when restricted to specific subclasses of configurations.
\end{abstract}

\section{INTRODUCTION}

At moderate temperatures, atoms tend to form bonds and cluster together forming molecules and crystals. The actual 3d arrangement of atoms in such clusters has a fundamental influence on its physical and mechanical properties. Correspondingly, the prediction of such $3 \mathrm{~d}$ geometries is of great importance and a central object of study in chemistry and molecular biology.

The quantum nature of atomic bonding makes the determination of $3 \mathrm{~d}$ molecular geometries from first principles unfeasible as the number of atoms scales up. A successful model, capable of describing the geometry of comparatively large atomic ensembles, is Molecular Mechanics [11, 20]. There, configurations are modeled as collections of atomic positions and types, to which a configurational energy is associated. Such energy is specified in terms of classical potentials and is phenomenological in nature. In particular, it usually features two-body interactions, minimized at some preferred bond length, as well as three-body terms, favoring specific angles between bonds [5, 29, 31].

In the case of identical atoms, under the classical Born-Oppenheimer approximation, the configuration is purely determined by the atomic positions $\left\{\boldsymbol{x}_{1}, \ldots, \boldsymbol{x}_{n}\right\} \in \mathbb{R}^{3 n}$, and the configurational energy $\mathcal{E}=\mathcal{E}\left(\left\{\boldsymbol{x}_{1}, \ldots, \boldsymbol{x}_{n}\right\}\right)$. The latter is assumed to be decomposed as $\mathcal{E}=\mathcal{E}_{2}+\mathcal{E}_{3}$, where $\mathcal{E}_{2}$ and $\mathcal{E}_{3}$ correspond to two- and three-body contributions, respectively. In particular, $\mathcal{E}_{2}$ is a function of those pairs $\left(\boldsymbol{x}_{i}, \boldsymbol{x}_{j}\right)$ corresponding to bonded atoms, and $\mathcal{E}_{3}$ depends on those triplets $\left(\boldsymbol{x}_{i}, \boldsymbol{x}_{j}, \boldsymbol{x}_{k}\right)$ corresponding to the angle formed by the bonds $\left(\boldsymbol{x}_{i}, \boldsymbol{x}_{j}\right)$ and $\left(\boldsymbol{x}_{j}, \boldsymbol{x}_{k}\right)$. Having in mind the specific case of covalent and ionic bonding, we will neglect long-range interactions in the following. In particular, $\mathcal{E}$ will feature nearest- and (partially) next-to-nearest interaction only.

2010 Mathematics Subject Classification. 52C25, 92E10.

Key words and phrases. Configurations in $\mathbb{Z}^{2}$, angle-rigidity, deformations, local minimizers. 
A central issue with respect to molecular structuring is that of stability: actual $3 \mathrm{~d}$ molecular arrangements need to show some stability (thermodynamic, electrochemical, mechanical) in order to be observed in experiments. In the context of Molecular Mechanics, this calls for identifying strict (local or global) minimizers of the configurational energy. In the setting of two- and three-body interactions described above, two configurations with identical bond lengths and bond angles necessarily have the same energy. As such, in order to classify strict energy minimizers, one is asked to identify those configurations which cannot be infinitesimally perturbed (up to isometries) without changing bond lengths or bond angles. We call these configurations angle-rigid, inspired by related rigidity concepts in graph theory, see Section 2.2 below. Note that the concept of angle-rigidity has a clear molecular-mechanical relevance, for the occurrence of different molecular geometries having same bond lengths and angles is not uncommon and is usually referred to as conformational isomerism. Butane $\left(\mathrm{C}_{4} \mathrm{H}_{10}\right)$ is a classical example featuring more possible conformal isomers [1].

The focus of this article is to investigate angle-rigidity for a specific class of $3 \mathrm{~d}$ configurations inspired by crystallization [4, see Figure 1. These are given as finite collections

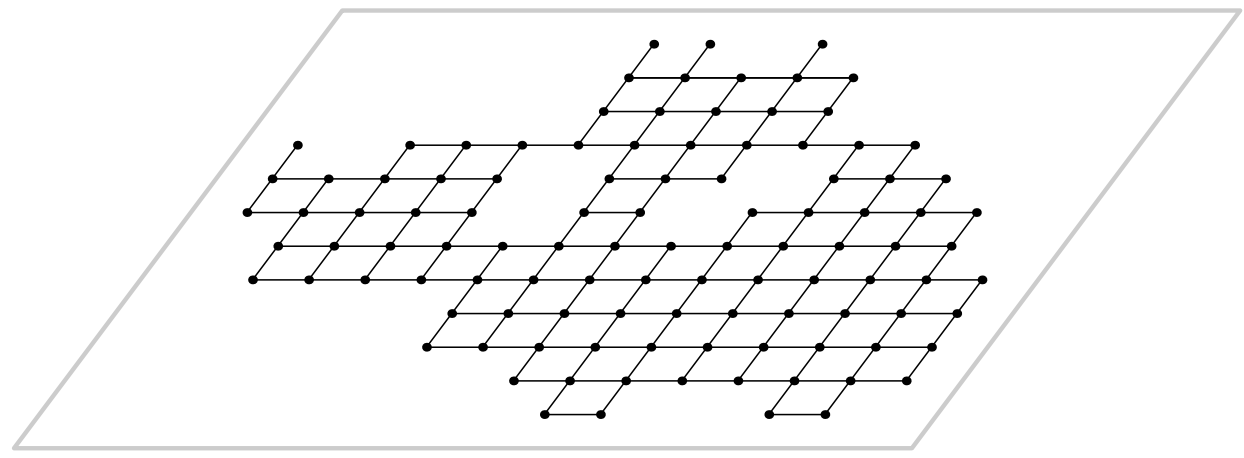

Figure 1. A $\mathbb{Z}^{2}$ configuration

of points in $\mathbb{Z}^{2} \times\{0\}$, with bonds corresponding to first neighbors. We advance a sufficient condition (6) for strict minimality, based on a straightforward combinatorial argument. Such sufficient condition (6) is in general not necessary, but turns out to fully characterize strict minimizers when restricted to particular classes of configurations. We call such classes foldings and shear-resistant configurations, inspired by the specific geometry of their respective perturbations which do not change bonds lengths nor bond angles. Note that these two classes do not cover all configurations: there are non-strict minimizing configurations which are neither foldings nor shear-resistant. On the other hand, every strict minimizing configurations is shear-resistant and thus covered by the characterization (see also Figure 7). The relevance of our results in connection with applications in Molecular Mechanics is discussed in Section 3 below.

The paper is organized as follows. In Section 2 we introduce notation and basic concepts. Although our focus is on strict minimality of configurations, the setting of our problem is strongly reminiscent of rigidity in graph theory. We comment on this 
relation in Section 2.2. The characterization of strict minimizers of two- and threebody-interaction configurational energies in terms of angle-rigidity is given in Section 3 . The sufficient condition for non-angle-rigidity is presented in Section 4, together with the discussion of folding configurations. Sections 546 then discuss the fine geometry of shear-resistance configurations.

\section{Definition of Angle-Rigid Configurations}

This section is devoted to introducing some basic notions and notation, leading to the Definition 2.1 of angle-rigid $\mathbb{Z}^{2}$ configurations. In Section 2.2 we collect some comments on the relation of this notion with other rigidity concepts in graph theory.

2.1. Basic notation and definition of angle-rigid $\mathbb{Z}^{2}$ configurations. Our analysis focuses on point configurations in three space dimensions, namely on collections $C=$ $\left\{\boldsymbol{x}_{k}\right\}_{k=1}^{n}$ of $n$ distinct points in $\mathbb{R}^{3}$. Among these, we call $\mathbb{Z}^{2}$ configurations those with

$$
\boldsymbol{x}_{k} \in \mathbb{Z}^{2}=\{(i, j, 0): i, j \in \mathbb{Z}\}, \quad \text { for all } k \in\{1, \ldots, n\},
$$

see Figure 1, and indicate them as $C \in \mathbb{Z}^{2 n}$ in the following. Note that we interpret $\mathbb{Z}^{2}$ as a proper subset of $\mathbb{R}^{3}$. In order to shorten notation, whenever possible we refer to points in $\mathbb{Z}^{2}$ by specifying their first two integer coordinates only. We call axes the subsets of $\mathbb{Z}^{2}$ of the form $\{i\} \times \mathbb{Z}$ or $\mathbb{Z} \times\{j\}$, for some $i, j \in \mathbb{Z}$.

To each configuration $C=\left\{\boldsymbol{x}_{k}\right\}_{k=1}^{n}$ of $n$ points in $\mathbb{R}^{3}$, we associate the segments joining pairs of points having distance smaller than $1+2 \varepsilon$, for some given and fixed small parameter $0<\varepsilon \leq(\sqrt{2}-1) / 4$. More precisely, we associate to $C$ the set of neighbors as specified by the pairs

$$
N(C)=\left\{\left(k, k^{\prime}\right) \in\{1, \ldots, n\}^{2}:\left|\boldsymbol{x}_{k}-\boldsymbol{x}_{k^{\prime}}\right| \leq 1+2 \varepsilon\right\} .
$$

Note that each pair of neighbors is counted twice in $N(C)$. If $\left(k, k^{\prime}\right) \in N(C)$, we call the straight segment joining $\boldsymbol{x}_{k}$ and $\boldsymbol{x}_{k^{\prime}}$ a bond and we say that the two points are bonded. Bonds are hence identified with pairs $\left(k, k^{\prime}\right) \in N(C)$, up to permutations. For each $\mathbb{Z}^{2}$ configuration one has that $\left(k, k^{\prime}\right) \in N(C)$ iff $\boldsymbol{x}_{k}$ and $\boldsymbol{x}_{k^{\prime}}$ are at distance 1 . In the following, we will use the notation $\boldsymbol{N}(C)=\left\{\left(\boldsymbol{x}_{k}, \boldsymbol{x}_{k^{\prime}}\right):\left(k, k^{\prime}\right) \in N(C)\right\}$ to indicate the pairs of bonded points.

We also indicate adjacent bonds by defining the set of triplets

$$
T(C)=\left\{\left(k, k^{\prime}, k^{\prime \prime}\right) \in\{1, \ldots, n\}^{3}:\left(k, k^{\prime}\right),\left(k^{\prime}, k^{\prime \prime}\right) \in N(C), k \neq k^{\prime \prime}\right\} .
$$

To each triplet $\left(k, k^{\prime}, k^{\prime \prime}\right) \in T(C)$, we uniquely associate the angle $\theta\left(\boldsymbol{x}_{k}, \boldsymbol{x}_{k^{\prime}}, \boldsymbol{x}_{k^{\prime \prime}}\right)$ formed by the vectors $\boldsymbol{x}_{k}-\boldsymbol{x}_{k^{\prime}}$ and $\boldsymbol{x}_{k^{\prime \prime}}-\boldsymbol{x}_{k^{\prime}}$ and oriented clockwise. Note that $\theta\left(\boldsymbol{x}_{k}, \boldsymbol{x}_{k^{\prime}}, \boldsymbol{x}_{k^{\prime \prime}}\right)+$ $\theta\left(\boldsymbol{x}_{k^{\prime \prime}}, \boldsymbol{x}_{k^{\prime}}, \boldsymbol{x}_{k}\right)=2 \pi$. We use the notation $\boldsymbol{T}(C)=\left\{\left(\boldsymbol{x}_{k}, \boldsymbol{x}_{k^{\prime}}, \boldsymbol{x}_{k^{\prime \prime}}\right):\left(k, k^{\prime}, k^{\prime \prime}\right) \in T(C)\right\}$ to indicate triplets of bonded points in the configuration.

We call the collection of all bonds of a configuration $C$ the bond structure of $C$. A collection of pairwise distinct points $\left(\boldsymbol{x}_{1}, \ldots, \boldsymbol{x}_{m}\right)$ in $C$ is called a path if $(i, i+1) \in N(C)$ for $i=1, \ldots, m-1$. Moreover, we say $\left(\boldsymbol{x}_{1}, \ldots, \boldsymbol{x}_{m}\right)$ is a cycle if it is a path and $(m, 1) \in N(C)$. By the Jordan curve theorem, each planar cycle which is a closed loop without self-intersection partitions its support plane into an interior and an exterior. 
We say that a configuration $C$ is connected if each two points of $C$ can be connected by a path. In a similar fashion, we define connected components of configurations.

For $C \in \mathbb{Z}^{2 n}$, denoting by $B \subset \mathbb{R}^{2}$ the union of the bonds, i.e., the union of the line segments between $\boldsymbol{x}_{k}$ and $\boldsymbol{x}_{k^{\prime}}$ for $\left(k, k^{\prime}\right) \in N(C)$, we call the bounded connected components of the open set $\mathbb{R}^{2} \backslash B$ the faces of $C$. For each face $f$, we call the points in $C$ on the boundary of the face a cell. More precisely, given $f$, we define $Z=\partial f \cap C$, and write $f(Z)$ to highlight that $f(Z)$ is the face associated to the cell $Z$. The collection of all cells of $C$ is denoted by $\mathcal{Z}(C)$. Note that in general each point may be contained in different cells.

Let now $\widetilde{C}=\left\{\tilde{\boldsymbol{x}}_{1}, \ldots, \tilde{\boldsymbol{x}}_{n}\right\} \in \mathbb{R}^{3 n}$ be an $\varepsilon$-perturbation of the configuration $C \in \mathbb{Z}^{2 n}$, namely, $\left|\boldsymbol{x}_{k}-\tilde{\boldsymbol{x}}_{k}\right|<\varepsilon$ for all $k \in\{1, \ldots, n\}$. Owing to definition (1) and $0<\varepsilon \leq$ $(\sqrt{2}-1) / 4$, we have that $N(\widetilde{C})=N(C)$ and $T(\widetilde{C})=T(C)$. In other words, the topology of the bond structure of $C$ and $\widetilde{C}$ are the same. We can now pose the following.

Definition 2.1 (Angle-rigid $\mathbb{Z}^{2}$ configurations). We say that a configuration $C \in \mathbb{Z}^{2 n}$ is angle-rigid if there exists $\delta \in(0, \varepsilon)$ such that any configuration $\widetilde{C}=\left\{\tilde{\boldsymbol{x}}_{1}, \ldots, \tilde{\boldsymbol{x}}_{n}\right\} \in \mathbb{R}^{3 n}$ with $\left|\boldsymbol{x}_{k}-\tilde{\boldsymbol{x}}_{k}\right|<\delta$ for all $k=1, \ldots, n$ and

$$
\begin{aligned}
& \left|\tilde{\boldsymbol{x}}_{k}-\tilde{\boldsymbol{x}}_{k^{\prime}}\right|=\left|\boldsymbol{x}_{k}-\boldsymbol{x}_{k^{\prime}}\right| \quad \forall\left(k, k^{\prime}\right) \in N(C)=N(\widetilde{C}), \\
& \theta\left(\tilde{\boldsymbol{x}}_{k}, \tilde{\boldsymbol{x}}_{k^{\prime}}, \tilde{\boldsymbol{x}}_{k^{\prime \prime}}\right)=\theta\left(\boldsymbol{x}_{k}, \boldsymbol{x}_{k^{\prime}}, \boldsymbol{x}_{k^{\prime \prime}}\right) \quad \forall\left(k, k^{\prime}, k^{\prime \prime}\right) \in T(C)=T(\widetilde{C}),
\end{aligned}
$$

is congruent to $C$, namely, $\left|\tilde{\boldsymbol{x}}_{k}-\tilde{\boldsymbol{x}}_{k^{\prime}}\right|=\left|\boldsymbol{x}_{k}-\boldsymbol{x}_{k^{\prime}}\right|$ for all $k, k^{\prime}=1, \ldots, n$.

The above definition can be equivalently presented in more mechanical terms. Let us call a mapping $\boldsymbol{\varphi}: C \rightarrow \mathbb{R}^{3 n}$ angle-preserving if $\tilde{C}:=\left\{\boldsymbol{\varphi}\left(\boldsymbol{x}_{1}\right), \ldots, \boldsymbol{\varphi}\left(\boldsymbol{x}_{n}\right)\right\}$ is an $\varepsilon$-perturbation of $C$ and

$$
\begin{aligned}
& \left|\boldsymbol{\varphi}\left(\boldsymbol{x}_{k}\right)-\boldsymbol{\varphi}\left(\boldsymbol{x}_{k^{\prime}}\right)\right|=1 \quad \forall\left(k, k^{\prime}\right) \in N(C), \\
& \theta\left(\boldsymbol{\varphi}\left(\boldsymbol{x}_{k}\right), \boldsymbol{\varphi}\left(\boldsymbol{x}_{k^{\prime}}\right), \boldsymbol{\varphi}\left(\boldsymbol{x}_{k^{\prime \prime}}\right)\right)=\theta\left(\boldsymbol{x}_{k}, \boldsymbol{x}_{k^{\prime}}, \boldsymbol{x}_{k^{\prime \prime}}\right) \quad \forall\left(k, k^{\prime}, k^{\prime \prime}\right) \in T(C) .
\end{aligned}
$$

A configuration $C \in \mathbb{Z}^{2 n}$ is then angle-rigid iff there exists $\delta \in(0, \varepsilon)$ such that all angle preserving maps $\boldsymbol{\varphi}$ with $\left|\boldsymbol{x}_{k}-\boldsymbol{\varphi}\left(\boldsymbol{x}_{k}\right)\right|<\delta$ for all $k=1, \ldots, n$ are (restrictions to $C$ of) isometries.

In the sequel, mappings $\varphi$ which are isometries (restricted to $C$ ) will be called trivial, otherwise nontrivial. As already mentioned in the Introduction, angle-preserving mappings that keep the points coplanar are trivial. Therefore, nontrivial angle-preserving mappings necessarily have a nonplanar image. The focus of the forthcoming Sections 46 is to discuss a sufficient and a conditional necessary condition for angle-rigidity which can be checked with limited computational effort.

2.2. Relation with rigidity in graphs. In the whole paper, we use a notation adapted to our mechanical application, by focusing on point configurations instead of the graph structure of bonds. This is in particular tailored to the check of strict minimality of configurational energies, as discussed in Section 3 below.

Before moving on, we would like to mention, however, that our setting is closely related to notions in graph-rigidity theory [3, 19, 22]. In fact, the notation above 
corresponds to associating to each $\mathbb{Z}^{2}$ configuration $C=\left\{\boldsymbol{x}_{k}\right\}_{k=1}^{n} \in \mathbb{Z}^{2 n}$ the unit-disk graph $G=(V, E)$, namely, a graph with one vertex for each point in $C$, and with an edge between two vertices whenever the corresponding points in $C$ are at distance 1 [14]. In particular, the set of edges $E$ can be specified as

$$
E=\left\{\left(k, k^{\prime}\right) \in V \times V:\left|\boldsymbol{x}_{k}-\boldsymbol{x}_{k^{\prime}}\right|=1\right\} .
$$

A framework is then a pair $(G, p)$, where $p: G \rightarrow \mathbb{R}^{3}$ is referred to as the realization of the graph $G$. We canonically associate to $C$ the framework $(G, p)$ given by $p(k):=\boldsymbol{x}_{k}$ for all $k \in V$. We may also indicate triplets as

$$
T=\left\{\left(k, k^{\prime}, k^{\prime \prime}\right) \in V \times V \times V:\left(k, k^{\prime}\right),\left(k^{\prime}, k^{\prime \prime}\right) \in E, k \neq k^{\prime \prime}\right\}
$$

and associate to each triplet $\left(k, k^{\prime}, k^{\prime \prime}\right) \in T$ the angle $\theta\left(p(k), p\left(k^{\prime}\right), p\left(k^{\prime \prime}\right)\right)$ formed by the vectors $p(k)-p\left(k^{\prime}\right)$ and $p\left(k^{\prime \prime}\right)-p\left(k^{\prime}\right)$ and oriented clockwise. We note that $E$ and $T$ coincide with $N(C)$ and $T(C)$ defined in (1) and (2), respectively, provided that we let $V=\{1, \ldots, n\}$.

A framework is said to be rigid if there exists $\delta>0$ such that all frameworks $(G, q)$ with $|p(k)-q(k)|<\delta$ for all $k \in V$ with the property

$$
\left|q(k)-q\left(k^{\prime}\right)\right|=\left|p(k)-p\left(k^{\prime}\right)\right| \quad \forall\left(k, k^{\prime}\right) \in E
$$

are congruent to $(G, p)$, namely, $\left|q(k)-q\left(k^{\prime}\right)\right|=\left|p(k)-p\left(k^{\prime}\right)\right|$ for all $\left(k, k^{\prime}\right) \in V \times V$. We refer the Reader to [27] for details about rigidity of frameworks.

Here, we add the restriction on the preservation of the angles and call angle-rigid a framework $(G, p)$ if there exists $\delta>0$ such that all frameworks $(G, q)$ with $|p(k)-q(k)|<$ $\delta$ for all $k \in V$ with the properties

$$
\begin{aligned}
& \left|q(k)-q\left(k^{\prime}\right)\right|=\left|p(k)-p\left(k^{\prime}\right)\right| \quad \forall\left(k, k^{\prime}\right) \in E, \\
& \theta\left(q(k), q\left(k^{\prime}\right), q\left(k^{\prime \prime}\right)\right)=\theta\left(p(k), p\left(k^{\prime}\right), p\left(k^{\prime \prime}\right)\right) \quad \forall\left(k, k^{\prime}, k^{\prime \prime}\right) \in T,
\end{aligned}
$$

are congruent to $(G, p)$.

Given the above mentioned identifications, a $\mathbb{Z}^{2}$ configuration $C$ is angle-rigid iff its associated framework $(G, p)$ is angle-rigid, so that these viewpoints are in fact equivalent. The graph theoretic notation may be more transparent in places. Still, in the very specific situation considered here, namely, that of $\mathbb{Z}^{2}$ configurations, it seems to be adding an unnecessary degree of abstraction. In fact, by directly referring to graphrigidity notions would complicate some of our arguments, which are based on the direct manipulation of the underlying configurations. We hence refrain from using graphrigidity notions in the following, focusing on point configurations instead.

Let us mention that rigidity under angle preservation has recently attracted attention. Applications arise from interacting systems of agents, as in robotics and traffic flow [2, 25]. Note that the term angle-rigid has in fact already been used in [7, although in a different, purely planar context. See also [6, 12, 13, 16, 26, 28] for some other results in the plane, where angles come into play. In the specific case of molecular structures, the celebrated Molecular Conjecture [30] relates to the possibility of investigating the rigidity of so-called molecular graphs [15] by combinatorial methods. 
Our notion of angle-rigidity is strictly related to that of body-and-hinge frameworks [18. These are collections of $d$-dimensional rigid bodies connected by hinges, where a hinge is identified with a $(d-2)$-dimensional affine subspace. Continuous motions of the body-and-hinge framework are allowed under the constraints that distances between two connected bodies are preserved and that their relative motion is a rotation around the common hinge. A body-and-hinge framework is said to be rigid if every such admissible motion is an isometry. To each $\mathbb{Z}^{2}$ configuration $\left\{\boldsymbol{x}_{1}, \ldots, \boldsymbol{x}_{n}\right\}$ one can associate a bodyand-hinge framework by considering a small $3 \mathrm{~d}$ sphere centered at each $\left\{\boldsymbol{x}_{1}, \ldots, \boldsymbol{x}_{n}\right\}$ and a hinge for each bond. Under this identification, a $\mathbb{Z}^{2}$ configuration is angle-rigid iff its associated body-and-hinge framework is rigid. Note however that the two notions are not equivalent, for a body-and-hinge framework made of two hinged rigid bodies which are not rotationally symmetric around the hinge is not rigid, whereas each $\mathbb{Z}^{2}$ connected configuration made of two points is angle-rigid. Combinatorial characterizations of the infinitesimal rigidity for $d$-dimensional body-and-hinge frameworks are indeed available [18. These pave the ground for investigating the angle-rigidity of molecules by welldeveloped tree algorithms on the underlying graphs.

Our setting is here more restricted, for it exclusively applies to the special class of $\mathbb{Z}^{2}$ configurations. Within this specific setting, we are able to present a characterization of framework rigidity. In comparison with [18, our characterization has the advantage of making the check of angle-rigidity straightforward, for the ensuing non-angle-rigidity sufficient condition (6) is elementarily implemented. We expect that similar techniques can be applied to other special classes, possibly including unit disk graphs generated by subsets of the triangular or the hexagonal lattice.

\section{STRICT LOCAL MiNimality AND ANGLE-RIGIDITY}

Before moving to the investigation of angle-rigidity for $\mathbb{Z}^{2}$ configurations, we comment here on the main application of our theory, namely the characterization of locally stable point configurations.

Assume to be given a configurational energy $\mathcal{E}: \mathbb{R}^{3 n} \rightarrow[0,+\infty]$ associating to each configuration $C=\left\{\boldsymbol{x}_{k}\right\}_{k=1}^{n} \in \mathbb{R}^{3 n}$ the scalar value $\mathcal{E}(C)$ given by

$$
\mathcal{E}(C)=\mathcal{E}_{2}(C)+\mathcal{E}_{3}(C):=\frac{1}{2} \sum_{N(C)} v_{2}\left(\left|\boldsymbol{x}_{k}-\boldsymbol{x}_{k^{\prime}}\right|\right)+\frac{1}{2} \sum_{T(C)} v_{3}\left(\theta\left(\boldsymbol{x}_{k}, \boldsymbol{x}_{k^{\prime}}, \boldsymbol{x}_{k^{\prime \prime}}\right)\right) .
$$

The configurational energy is the sum of a two-body term $\mathcal{E}_{2}$, depending solely on the mutual distance of the points, and a three-body contribution $\mathcal{E}_{3}$ depending on angles instead. The two-body interaction density $v_{2}:[0, \infty) \rightarrow[0,+\infty]$ is assumed to be strictly minimized at the reference distance 1 with $v_{2}(1)=0$. The three-body interaction energy $\mathcal{E}_{3}$ is modulated via the three-body interaction density $v_{3}:[0,2 \pi] \rightarrow[0, \infty)$ which we assume to be symmetric around $\pi$ and to attain its minimal value 0 just at $\pi / 2, \pi$, and $3 \pi / 2$. Note that the factors $1 / 2$ account for double counting neighbors in $N(C)$ and triplets in $T(C)$. Without claiming completeness, the Reader is referred to the seminal papers [5, 29, 31] for the discussion of empirical potentials including three-body contributions and to [8, 9, 10, 23, 24] for some related mathematical crystallization 
results. The Reader is also referred to [17, 18, 32] for a collection of graph-theoretical results of relevance in Molecular Mechanics.

We stress that all $\mathbb{Z}^{2}$ configurations $C$ are global minimizers of the energy, as one has $\mathcal{E}(C)=0$. We then ask ourselves if these configurations are strict local minimizers or not, where local minimality is understood with respect to the Euclidean norm in $\mathbb{R}^{3 n}$. Local minimality is indeed crucial in connection with applications, for it formalizes the concept of stability of discrete structures. The relation of strict local minimality and angle-rigidity is given by the following.

Proposition 3.1 (Angle-rigidity = strict local minimality). A configuration is a strict local minimizer for the energy $\mathcal{E}$ up to isometries iff it is angle-rigid.

Proof. Let $C=\left\{\boldsymbol{x}_{k}\right\}_{k=1}^{n} \in \mathbb{Z}^{2 n}$ be angle-rigid with given $\delta \in(0, \varepsilon)$ and let $\widetilde{C}=$ $\left\{\tilde{\boldsymbol{x}}_{1}, \ldots, \tilde{\boldsymbol{x}}_{n}\right\} \in \mathbb{R}^{3 n}$ with $\left|\boldsymbol{x}_{k}-\tilde{\boldsymbol{x}}_{k}\right|<\delta$ for all $k=1, \ldots, n$ such that $C$ and $\widetilde{C}$ are not congruent. Hence, there exists either $\left(k, k^{\prime}\right) \in N(C)$ such that $\left|\tilde{\boldsymbol{x}}_{k}-\tilde{\boldsymbol{x}}_{k^{\prime}}\right| \neq 1$ or there exists $\left(k, k^{\prime}, k^{\prime \prime}\right) \in T(\widetilde{C})$ such that $\theta\left(\tilde{\boldsymbol{x}}_{k}, \tilde{\boldsymbol{x}}_{k^{\prime}}, \tilde{\boldsymbol{x}}_{k^{\prime \prime}}\right) \notin\{\pi / 2, \pi, 3 \pi / 2\}$ (or both). In all cases, $\mathcal{E}(\widetilde{C})>0=\mathcal{E}(C)$, which proves that $C$ is a strict local minimizer of $\mathcal{E}$ in a $\delta$-neighborhood, up to isometries.

Let now $C$ be not angle-rigid. Then, for all $\delta<\varepsilon$ there exists $\widetilde{C}_{\delta}=\left\{\tilde{\boldsymbol{x}}_{1}, \ldots, \tilde{\boldsymbol{x}}_{n}\right\} \in$ $\mathbb{R}^{3 n}$ with $\left|\boldsymbol{x}_{k}-\tilde{\boldsymbol{x}}_{k}\right|<\delta$ for all $k=1, \ldots, n$ such that $C$ and $\widetilde{C}_{\delta}$ are not congruent, $\left|\tilde{\boldsymbol{x}}_{k}-\tilde{\boldsymbol{x}}_{k^{\prime}}\right|=1$ for all $\left(k, k^{\prime}\right) \in N\left(\widetilde{C}_{\delta}\right)$, and $\theta\left(\tilde{\boldsymbol{x}}_{k}, \tilde{\boldsymbol{x}}_{k^{\prime}}, \tilde{\boldsymbol{x}}_{k^{\prime \prime}}\right) \in\{\pi / 2, \pi, 3 \pi / 2\}$ for all $\left(k, k^{\prime}, k^{\prime \prime}\right) \in T\left(\widetilde{C}_{\delta}\right)$. Hence, $\mathcal{E}\left(\widetilde{C}_{\delta}\right)=\mathcal{E}(C)=0$ and $C$ is not a strict local minimizer.

We conclude this section by commenting on the relevance of this stability result with respect to applications. Our setting is admittedly restricted, for the configurational energy $\mathcal{E}$ solely features nearest-neighbors interactions, together with some aspects of next-to-nearest-neighbors interactions. In particular, long-range interactions are not considered, which is surely a limitation. Note however that our energy is tailored to the modeling of ionic and covalent bonding at very low temperature. In this setting, the effect of long-range interactions on the structure is usually negligible. This is for instance the case in honeycomb carbon ( $\mathrm{sp}^{2}$-hybridization), where bonds range from $1.39 \pm 0.02, \AA$ for benzene to $1.42 \AA$ for graphene, thus differring by $2 \%$.

In addition, including long-range interactions in the model would contribute another source of rigidity, due to long-range effects. We believe that it would make the understanding of the interplay between geometry and rigidity less transparent. Let us moreover mention that rigorous crystallization results taking long-range interactions into account are scarce and usually very specific with respect to the choice of the interaction potentials. To the best of our knowledge, no long-range crystallization result for $2 \mathrm{~d}$ cubic systems at zero temperature is available. On the contrary, such crystallization result is available in our nearest- and next-to-nearest-neighbors setting [23].

The choice of investigating $\mathbb{Z}^{2}$ in $3 \mathrm{~d}$ is also very specific, and not directly related to any specific $2 \mathrm{~d}$ atomic system. Our choice is here motivated by simplicity. We expect that similar results could be obtained for other crystalline $2 \mathrm{~d}$ geometries, as well, including for instance the regular triangular lattice, the regular hexagonal lattice, and the general 
Bravais lattice. This would however call for some substantial notational and technical changes, due to the distinct symmetries of the $2 \mathrm{~d}$ structures.

Note again that the energy is degenerate, for $\mathcal{E}=0$ on any configuration in $\mathbb{Z}^{2}$. This crude idealization is motivated by our interest in the phenomenon of conformational isomerism [1, where bond-rotation phenomena occur. Here, energy barriers between alternative configurations, although being not zero, are usually small $\left(10^{0} \sim 10^{1} \mathrm{kcal} / \mathrm{mol}\right)$ in comparison with ionic bonding energy $\left(10^{2} \sim 10^{3} \mathrm{kcal} / \mathrm{mol}\right)[21$.

\section{Sufficient condition: Combinatorial Characterization of Foldings}

All disconnected configurations are obviously not angle-rigid. We will hence concentrate on connected configurations from now on. Checking angle-rigidity for connected configurations directly from the definition may be very demanding. We are thus interested in reducing the complexity of the problem by presenting a more tractable condition implying non-angle-rigidity. Recall that we call axes the sets $\{i\} \times \mathbb{Z} \subset \mathbb{Z}^{2}$ or $\mathbb{Z} \times\{i\} \subset \mathbb{Z}^{2}$, for some $i \in \mathbb{Z}$. In this section we prove the following sufficient condition.

Proposition 4.1 (Sufficient condition). Let $C \in \mathbb{Z}^{2 n}$ be connected and fulfill the following condition:

there exists an axis $A$ such that, by removing from $C$ all points on $A$ having

at most one neighbor off $A$, the resulting configuration is disconnected.

Then, $C$ is not angle-rigid.

Condition (6) is illustrated in Figure 2, Checking condition (6) for some general configuration $C \in \mathbb{Z}^{2 n}$ can be accomplished very efficiently. Indeed, one has to consider all axes intersecting $C$ (at most $2 n$ ), remove points according to (6), and check for connectedness of the resulting configuration.

The proof of Proposition 4.1 relies on an equivalent geometric characterization of condition (6) in terms of a specific class of configurations, so-called foldings $\mathcal{F}$.

Definition 4.2 (Folding). We say that a configuration $C \in \mathbb{Z}^{2 n}$ is a folding, and we write $C \in \mathcal{F}$, if there exists an axis $A$ and a subconfiguration $\widetilde{C} \subset C$ such that both $\widetilde{C} \backslash A$ and $(C \backslash \widetilde{C}) \backslash A$ are not empty and, letting $R_{\rho}$ be a rotation about $A$ of amplitude $\rho$ with $\rho \in(0, \pi / 2)$ arbitrarily small, the map $\varphi: C \rightarrow \mathbb{R}^{3 n}$ defined as

$$
\boldsymbol{\varphi}(\boldsymbol{x})= \begin{cases}R_{\rho}(\boldsymbol{x}) & \text { if } \boldsymbol{x} \in \widetilde{C} \\ \boldsymbol{x} & \text { if } \boldsymbol{x} \in C \backslash \widetilde{C}\end{cases}
$$

is angle-preserving, i.e., fulfills (3)-(4).

We have the following characterization.

Proposition 4.3 (Characterization). $C \in \mathcal{F} \Leftrightarrow(6)$ holds.

In other words, condition (6) can be understood as a combinatorial characterization of $\mathcal{F}$. Of course, all foldings are not angle-rigid since the map $\varphi$ from Definition 4.2 is angle-preserving and nontrivial. Consequently, Proposition 4.3 immediately implies Proposition 4.1. We now prove Proposition 4.3. 

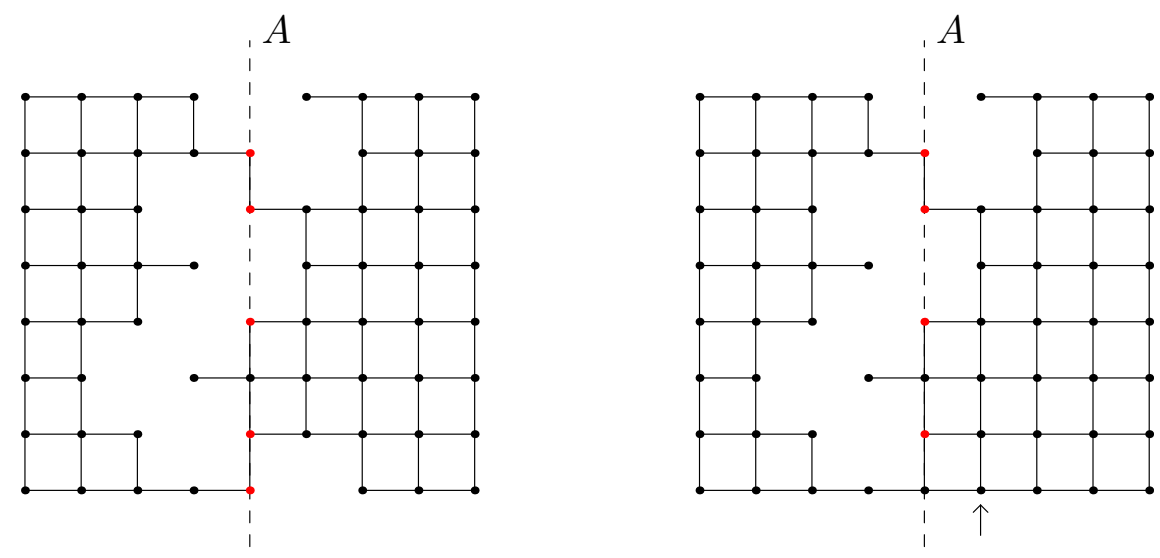

FIgURE 2. Illustration of condition (6). In both configurations, points on the axis $A$ having at most one neighbor off $A$ are highlighted. Upon removing them, the left configuration becomes disconnected (i.e., condition (6) holds) whereas the right configuration is still connected (condition (6) does not hold, respectively). Note that the two configurations differ by the point indicated by the arrow.

Proof. Step 1: Implication $(6) \Rightarrow C \in \mathcal{F}$. Let $C$ be connected and fulfill (6). Call $\widetilde{C}$ the configuration obtained by removing all points of $C$ belonging to $A$ and having at most one neighbor off $A$. Fix a connected component $C_{1} \subset \widetilde{C}$ of $\widetilde{C}$. Then, $C_{1}$ and $\widetilde{C} \backslash C_{1}$ are disconnected. Let $R_{\rho}$ represent a rotation around the axis $A$ of amplitude $\rho$, with $\rho \in(0, \pi / 2)$ small, and define the mapping $\varphi: C \rightarrow \mathbb{R}^{3 n}$ as

$$
\boldsymbol{\varphi}(\boldsymbol{x})=\left\{\begin{array}{lll}
R_{\rho}(\boldsymbol{x}) & \text { if } \boldsymbol{x} \in C_{1} \\
\boldsymbol{x} & \text { if } \boldsymbol{x} \in C \backslash C_{1} .
\end{array}\right.
$$

As $C_{1}$ and $\widetilde{C} \backslash C_{1}$ are disconnected, the map $\varphi$ fulfills (3)-(4) when restricted to $\widetilde{C}$.

We aim at showing that, by reintegrating the points in $C \backslash \widetilde{C}$ which were removed under condition (6), relations (3)-(4) still hold. This will show that $\varphi$ is angle-preserving, and therefore $C \in \mathcal{F}$. We proceed sequentially, by adding one point at a time. To this aim, assume to be given a configuration $\widehat{C}$ with $\widetilde{C} \subset \widehat{C} \subset C$ such that (3)-(4) hold for $\widehat{C}$ but $\widehat{C} \neq C$. Without loss of generality, we let $A=\mathbb{Z} \times\{0\}$ and $(0,0) \in C \backslash \widehat{C}$. We now check that, by adding the point $(0,0)$ to $\widehat{C}$, properties (3)-(4) still hold true. To prove this, we need to check that the new bonds and new angles that have to be included by considering the extra point $(0,0)$ are still invariant under $\boldsymbol{\varphi}$. Such new bonds and angles are determined by the pairs and the triplets

$$
\boldsymbol{N}^{\prime}:=\boldsymbol{N}(\widehat{C} \cup(0,0)) \backslash \boldsymbol{N}(\widehat{C}) \text { and } \boldsymbol{T}^{\prime}:=\boldsymbol{T}(\widehat{C} \cup(0,0)) \backslash \boldsymbol{T}(\widehat{C}),
$$

respectively. (We refer to Section 2.1 for the definition of $\boldsymbol{N}$ and $\boldsymbol{T}$.)

In view of condition $(6)$, as $(0,0) \notin \widetilde{C}$, we have that at least one of the points $(0,1)$ and $(0,-1)$ does not belong to $C$, and thus does not belong to $\widehat{C}$. In case both do not 
belong to $\widehat{C}$, the new bonds $\boldsymbol{N}^{\prime}$ are necessarily aligned with $A$, namely

$$
\boldsymbol{N}^{\prime} \subset\{((0,0),(1,0)),((1,0),(0,0)),((0,0),(-1,0)),((-1,0),(0,0))\} .
$$

Since $\boldsymbol{\varphi}$ is the identity on $A$, we have that $\left|\boldsymbol{\varphi}\left(\boldsymbol{x}_{k}\right)-\boldsymbol{\varphi}\left(\boldsymbol{x}_{k^{\prime}}\right)\right|=1$ for all $\left(\boldsymbol{x}_{k}, \boldsymbol{x}_{k^{\prime}}\right) \in \boldsymbol{N}^{\prime}$ as well. As regards triplets, we have that

$$
\left(\boldsymbol{x}_{k}, \boldsymbol{x}_{k^{\prime}}, \boldsymbol{x}_{k^{\prime \prime}}\right) \in \boldsymbol{T}^{\prime} \Rightarrow \text { at least two out of }\left\{\boldsymbol{x}_{k}, \boldsymbol{x}_{k^{\prime}}, \boldsymbol{x}_{k^{\prime \prime}}\right\} \text { belong to } A \text {. }
$$

As $\varphi$ is the identity on $A$ and either the identity or a rotation about $A$ out of $A$, one has that $\theta\left(\boldsymbol{\varphi}\left(\boldsymbol{x}_{k}\right), \boldsymbol{\varphi}\left(\boldsymbol{x}_{k^{\prime}}\right), \boldsymbol{\varphi}\left(\boldsymbol{x}_{k^{\prime \prime}}\right)\right)=\theta\left(\boldsymbol{x}_{k}, \boldsymbol{x}_{k^{\prime}}, \boldsymbol{x}_{k^{\prime \prime}}\right)$ for all $\left(\boldsymbol{x}_{k}, \boldsymbol{x}_{k^{\prime}}, \boldsymbol{x}_{k^{\prime \prime}}\right) \in \boldsymbol{T}^{\prime}$. In particular, $\varphi$ fulfills properties (3)-(4) on $\widehat{C} \cup(0,0)$.

Let us now consider the case $(0,1) \in \widehat{C}$ (the remaining case $(0,-1) \in \widehat{C}$ can be treated analogously). Here, all bonds in $\boldsymbol{N}^{\prime}$ are either aligned with $A$ as in (7), or orthogonal to $A$. In both cases, as $\varphi$ is the identity on $A$ and either the identity or a rotation about $A$ out of $A$, one has that $\left|\boldsymbol{\varphi}\left(\boldsymbol{x}_{k}\right)-\boldsymbol{\varphi}\left(\boldsymbol{x}_{k^{\prime}}\right)\right|=1$ for all $\left(\boldsymbol{x}_{k}, \boldsymbol{x}_{k^{\prime}}\right) \in \boldsymbol{N}^{\prime}$ as well. Those triplets in $\boldsymbol{T}^{\prime}$ containing at least two points on $A$ can be treated as before, see (8). We are just left to consider the triplets of the form

$$
((0,0),(0,1),(0,2)),((0,0),(0,1),(1,1)),((0,0),(0,1),(-1,1)),
$$

(and permutations $\left(\boldsymbol{x}_{k}, \boldsymbol{x}_{k^{\prime}}, \boldsymbol{x}_{k^{\prime \prime}}\right) \mapsto\left(\boldsymbol{x}_{k^{\prime \prime}}, \boldsymbol{x}_{k^{\prime}}, \boldsymbol{x}_{k}\right)$ ), if at all included in $\boldsymbol{T}^{\prime}$. Let us assume that $((0,0),(0,1),(1,1)) \in \boldsymbol{T}^{\prime}$. Then, $((0,1),(1,1)) \in \boldsymbol{N}(C)$, implying that $(0,1)$ and $(1,1)$ belong to the same connected component of $\widetilde{C}$. In case $(0,1),(1,1) \in \widetilde{C} \backslash C_{1}$ we have that $\varphi$ is the identity on $(0,0),(0,1)$, and $(1,1)$. In case $(0,1),(1,1) \in C_{1}$ we have that $\varphi$ is a rotation about $A$ on $(0,0),(0,1)$, and $(1,1)$. In both cases, $\varphi$ is an isometry on $(0,0),(0,1)$, and $(1,1)$, implying that

$$
\theta(\boldsymbol{\varphi}(0,0), \boldsymbol{\varphi}(0,1), \boldsymbol{\varphi}(1,1))=\theta((0,0),(0,1),(1,1)) .
$$

An analogous argument applies to $((0,0),(0,1),(0,2))$ and $((0,0),(0,1),(-1,1))$. Thus, we have proved that $\theta\left(\boldsymbol{\varphi}\left(\boldsymbol{x}_{k}\right), \boldsymbol{\varphi}\left(\boldsymbol{x}_{k^{\prime}}\right), \boldsymbol{\varphi}\left(\boldsymbol{x}_{k^{\prime \prime}}\right)\right)=\theta\left(\boldsymbol{x}_{k}, \boldsymbol{x}_{k^{\prime}}, \boldsymbol{x}_{k^{\prime \prime}}\right)$ for all $\left(\boldsymbol{x}_{k}, \boldsymbol{x}_{k^{\prime}}, \boldsymbol{x}_{k^{\prime \prime}}\right) \in$ $\boldsymbol{T}^{\prime}$. Eventually, also in case $(0,1) \in \widehat{C}$, the map $\varphi$ fulfills properties (3)-(4) on $\widehat{C} \cup(0,0)$.

In summary, we have shown that the map $\varphi$ fulfills properties (3)-(4) on $\widehat{C} \cup(0,0) \subset$ $C$. In case $\widehat{C} \cup(0,0) \neq C$, we repeat the argument by adding a point from $C \backslash(\widehat{C} \cup(0,0))$ to $\widehat{C} \cup(0,0)$, making sure that $\varphi$ preserves properties $(3)-(4)$. Since the number of points in $C$ is finite, this procedure eventually terminates, proving that $\varphi$ fulfills (3)-(4) on $C$. This shows that $\varphi$ is angle-preserving, and therefore $C \in \mathcal{F}$.

Step 2: Implication $C \in \mathcal{F} \Rightarrow(6)$. Consider $C \in \mathcal{F}$. Let $A$ and $\widetilde{C}$ be the axis and the subconfiguration from Definition 4.2 . Without loss of generality, possibly by changing coordinates, we can assume that $A=\mathbb{Z} \times\{0\}$.

The mapping $\varphi$ from Definition 4.2 does not preserve the length of bonds which connect points in $\widetilde{C}$ and $C \backslash \widetilde{C}$ and do not intersect the axis $A$. This implies that $\widetilde{C}$ and $C \backslash \widetilde{C}$ are connected just through bonds intersecting $A$.

We would like to prove that condition (6) holds with this same axis $A$. In order to do this, we shall prove that by removing from $C$ all points on $A$ having at most one neighbor off $A$, the resulting configuration $\widehat{C}$ is disconnected. 
Assume by contradiction that $\widehat{C}$ is connected. The two sets $\widetilde{C} \backslash A$ and $(C \backslash \widetilde{C}) \backslash A$ are nonempty and disjoint. Take a path in $\widehat{C}$ connecting a point in $\widetilde{C} \backslash A$ to a point in $(C \backslash \widetilde{C}) \backslash A$. As we have already seen that such path should contain bonds intersecting $A$, with no loss of generality, again by possibly redefining coordinates, we can find a path of the form

$$
\{(0,1),(0,0), \ldots,(k, 0),(k, \pm 1)\} \subset \widehat{C}
$$

where $k \in \mathbb{N} \cup\{0\}$ is given, $(0,1) \in \widetilde{C}$ and $(k, \pm 1) \in C \backslash \widetilde{C}$ (here, it is intended that either $(k, 1)$ or $(k,-1)$ belong to the path, and that for $k=0$ it is necessarily $(0,-1))$. As all points $(0,0), \ldots,(k, 0)$ belong to $\widehat{C}$ after the removal described in (6), one has that all points $(0, \pm 1), \ldots,(k, \pm 1)$ belong to $C$ as well. We first consider the case that $(k, 1) \in C \backslash \widetilde{C}$ is contained in the path. Then, as $(0,1) \in \widetilde{C}$, we find $0 \leq l \leq k-1$ such that $(l, 1) \in \widetilde{C}$ and $(l+1,1) \in C \backslash \widetilde{C}$. Hence, we have that

$$
|\boldsymbol{\varphi}(l, 1)-\boldsymbol{\varphi}(l+1,1)|=\left|R_{\rho}(l, 1)-(l+1,1)\right|>1
$$

contradicting (3). On the contrary, suppose that $(k,-1) \in C \backslash \widetilde{C}$ is contained in the path. If $(k, 1) \in C \backslash \widetilde{C}$, we find a contradiction as before. Thus, we can assume that $(k, 1) \in \widetilde{C}$. But then

$$
\theta(\boldsymbol{\varphi}(k, 1), \boldsymbol{\varphi}(k, 0), \boldsymbol{\varphi}(k,-1))=\theta\left(R_{\rho}(k, 1),(k, 0),(k,-1)\right) \neq \pi
$$

contradicting (4). We conclude that $\widehat{C}$ is necessarily disconnected and thus condition (6) holds.

\section{NeCESSARY CONDITION: SHEAR-RESISTANT CONFIGURATIONS $\mathcal{S}_{k}$}

In the previous section, we have seen that the sufficient condition $(6)$ is also necessary for non-angle-rigidity when restricting to the subclass of foldings. Let us point out, however, that (6) is not necessary in general, as the example in Figure 3 shows.

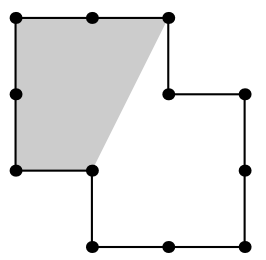

Figure 3. A not angle-rigid configuration not fulfilling (6). A nontrivial angle-preserving mapping $\varphi$ is given by $\boldsymbol{\varphi}(\boldsymbol{x})=\boldsymbol{x}$ at the boundary of the shaded region and $\boldsymbol{\varphi}(\boldsymbol{x})=\boldsymbol{x}+(0,1-\cos t, \sin t)$ elsewhere, for $t>0$ small.

The remainder of the paper is devoted to another class of configurations whose anglerigidity can be characterized via (6). These will be called $k$-shear-resistant, in coordination with an integer $k \in \mathbb{N}_{0}:=\mathbb{N} \cup\{0\}$, and will be denoted by $\mathcal{S}_{k}$.

Recall that, to each connected configuration $C \in \mathbb{Z}^{2 n}$, we can associate its bond structure. In particular, from Section 2.1 we recall the definition of the cells $Z \in \mathcal{Z}(C)$ 
of a configuration $C$, and the corresponding faces $f(Z)$. In the following, we say that a bond is acyclic if it is not contained in any simple cycle of the bond graph, i.e., in a closed loop without self-intersection.

We start by noting that acyclic bonds of a cell may influence angle-rigidity. More precisely, a configuration with acyclic bonds may not be angle-rigid but become anglerigid, when all acyclic bonds are removed, see the examples in Figure 4.

(a)
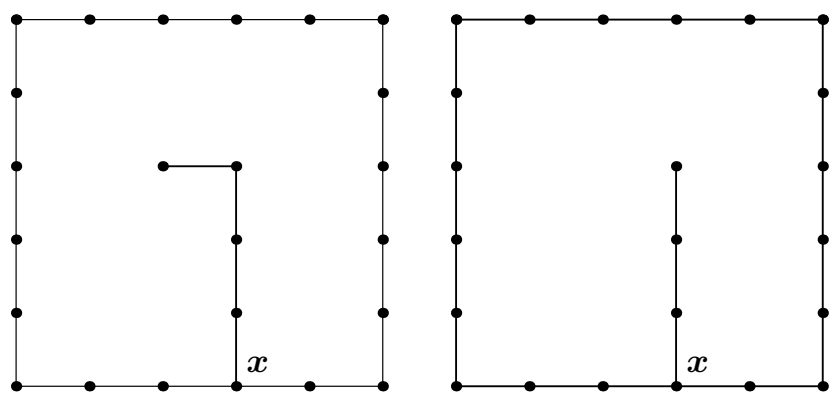

$\circ$ (c)

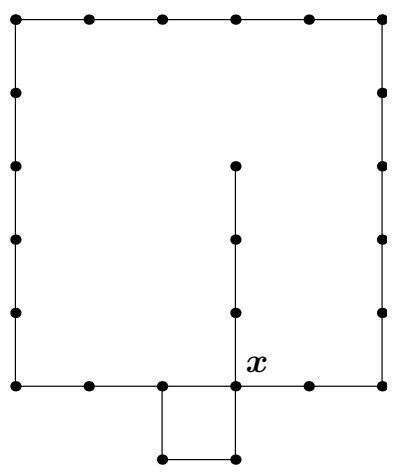

FIgURE 4. Illustration of not angle-rigid configurations. Configurations (a)-(b) get angle-rigid when acyclic bonds within the cells are removed, whereas configurations (c)-(d) remain not angle-rigid upon removal of the acyclic bonds.

In fact, the influence of acyclic bonds on angle-rigidity can be completely characterized in terms of the class $\mathcal{F}$, i.e., by condition (6). To this end, we consider a single connected components of acyclic bonds intersecting a simple cycle in $\boldsymbol{x}$. With reference to Figure 4, we have that: (a) If there does not exist an axis containing all acyclic bonds, then condition (6) holds for the vertical axis containing $\boldsymbol{x}$, and the configuration is not angle-rigid; (b) If acyclic bonds are aligned on an axis and $\boldsymbol{x}$ has only one bond on this axis, condition (6) holds for the horizontal axis containing the atom $\boldsymbol{x}$; (c) If $\boldsymbol{x}$ has two bonds on the axis, the angle-rigidity of the configuration is not affected by removing the acyclic bonds. Indeed, in view of (4), any angle-preserving mapping on the configuration obtained by removing the acyclic bonds can be uniquely extended to the original configuration; (d) If the acyclic bond connects two cycles, then condition (6) holds for the horizontal axis containing $\boldsymbol{y}$, and the configuration is not angle-rigid. Note that (a)-(d) exhaust all possibilities (up to multiple connected components of acyclic bonds). As we eventually aim at assessing if a configuration lies in $\mathcal{F} \cup \mathcal{S}_{k}$, we hence simplify the presentation from now on and assume with no further mention that there are no acyclic bonds.

Let $d$ denote the classical Manhattan distance in $\mathbb{Z}^{2}$, namely

$$
d(\boldsymbol{x}, \boldsymbol{y})=\left|\boldsymbol{x}_{1}-\boldsymbol{y}_{1}\right|+\left|\boldsymbol{x}_{2}-\boldsymbol{y}_{2}\right|
$$

for $\boldsymbol{x}=\left(x_{1}, x_{2}\right), \boldsymbol{y}=\left(y_{1}, y_{2}\right) \in \mathbb{Z}^{2}$. Moreover, let $d(\boldsymbol{x}, A)=\inf _{\boldsymbol{y} \in A} d(\boldsymbol{x}, \boldsymbol{y})$ for $\boldsymbol{x} \in \mathbb{Z}^{2}$ and $A \subset \mathbb{Z}^{2}$ nonempty. 
Given the cell $Z \in \mathcal{Z}(C)$ and $k \in \mathbb{N}_{0}$, we define the corresponding $k$-cell $Z^{(k)}$ by

$$
Z^{(k)}:=\{\boldsymbol{x} \in C \mid d(\boldsymbol{x}, Z) \leq k\}
$$

For each $k \in \mathbb{N}_{0}$, the collection of all $k$-cells $Z^{(k)}$ for $Z \in \mathcal{Z}(C)$ is denoted by $\mathcal{Z}^{(k)}(C)$. Note that $Z=Z^{(0)}$, so that cells are also called 0-cells in the following.

Four points $\boldsymbol{x}_{1}, \boldsymbol{x}_{2}, \boldsymbol{x}_{3}, \boldsymbol{x}_{4} \in \mathbb{Z}^{2}$ are said to form a paraxial rectangle if they are vertices of a rectangle in $\mathbb{Z}^{2}$ with sides aligned to axes in $\mathbb{Z}^{2}$ : namely, if $\boldsymbol{x}_{1}, \boldsymbol{x}_{2}, \boldsymbol{x}_{3}, \boldsymbol{x}_{4}$ can be obtained by translating via $\boldsymbol{z} \in \mathbb{Z}^{2}$ the points

$$
(0,0),(a, 0),(0, b),(a, b) \quad \text { for some } a, b \in \mathbb{N} \quad \text { with } \min \{a, b\}=1 .
$$

We are now ready to define $k$-shear-resistant configurations.

Definition 5.1 ( $k$-shear-resistant configurations). Let $C \in \mathbb{Z}^{2 n}$ be connected and let $k \in \mathbb{N}_{0}$.

(i) We say that a cell $Z \in \mathcal{Z}(C)$ is $k$-shear-resistant if, letting $Z^{(k)} \in \mathcal{Z}^{(k)}(C)$ be the corresponding $k$-cell, each angle-preserving mapping $\varphi: Z^{(k)} \rightarrow \mathbb{R}^{3 \# Z^{(k)}}$ has the following property: if two pairs $\left(\boldsymbol{x}_{i}, \boldsymbol{x}_{i^{\prime}}\right),\left(\boldsymbol{x}_{j}, \boldsymbol{x}_{j^{\prime}}\right) \in \boldsymbol{N}(Z)$ of pairwise distinct points form a paraxial rectangle whose interior lies entirely in $f(Z)$, then

$$
\varphi\left(x_{i}\right), \varphi\left(x_{i^{\prime}}\right), \varphi\left(x_{j}\right), \varphi\left(x_{j^{\prime}}\right)
$$

are coplanar.

(ii) We say that the configuration $C$ is $k$-shear-resistant, and we write $C \in \mathcal{S}_{k}$, if all its cells are $k$-shear-resistant.

The above definition implies that $\mathcal{S}_{k} \subset \mathcal{S}_{k^{\prime}}$ if $k \leq k^{\prime}$, for the set of nontrivial anglepreserving mappings of $Z^{(k)}$ decreases when $k$ increases. Moreover, every angle-rigid configuration consisting of $n$ points lies in $\mathcal{S}_{n}$. In Figure 5 we present examples and non-examples of $k$-shear-resistant configurations for various values of $k$.

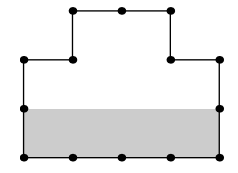

$C_{1} \notin \mathcal{S}_{0}$

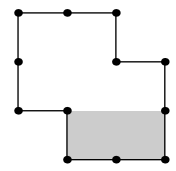

$C_{2} \notin \mathcal{S}_{0}$

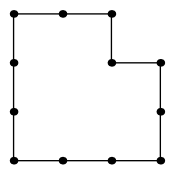

$C_{3} \in \mathcal{S}_{0}$

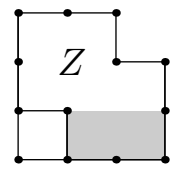

$C_{4} \in \mathcal{S}_{1} \backslash \mathcal{S}_{0}$

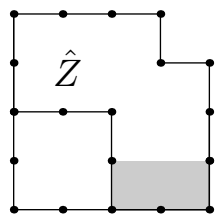

$C_{5} \in \mathcal{S}_{2} \backslash \mathcal{S}_{1}$

Figure 5. Examples of configurations which are (not) shear-resistant, cf. Section 6 below.

We emphasize that, for a cell $Z$, the property of being $k$-shear resistant depends not only on $Z$ itself but also on the configuration $C$. In particular, the same cell may be $k$-shear resistant as part of one configuration and not $k$-shear-resistant as part of another. Following the discussion of Figure 3, one has that the configuration $C_{2}$ in Figure 5 is not 0 -shear-resistant. Also $C_{1}$ in Figure 5 is not 0 -shear-resistant. Indeed, in Figure 3 we have described a nontrivial angle-preserving mapping of $C_{2}$ making the 
marked paraxial rectangle nonplanar. For $C_{1}$, we refer to the construction in the proof of Lemma 6.1 below, in particular to (17). We will give a complete characterization of 0 -shear-resistant configurations in Propositions 6.1 and 6.3 later on, which will imply in particular that $C_{3}$ in Figure 5 is 0-shear-resistant.

Cell $Z$ of configuration $C_{4}$ in Figure 5 is again $C_{2}$, hence not 0 -shear-resistant. As a consequence, $C_{4}$ is not 0 -shear-resistant. On the other hand, when considering $C_{4}=$ $Z^{(1)}$ the marked paraxial rectangle is forced to stay coplanar for any angle-preserving mapping. This in particular implies that $C_{4} \in \mathcal{S}_{1}$.

In the case of configuration $C_{5}$ in Figure 5 , one has that the top cell $\hat{Z}$ is not 0 -shearresistant. Any of its nontrivial angle-preserving mappings cannot keep the marked paraxial rectangle coplanar. Such transformations are not precluded by considering $\hat{Z}^{(1)}$ since by passing from $\hat{Z}$ to $\hat{Z}^{(1)}$ the configuration is only augmented by acyclic bonds. Thus, $C_{5}$ is not 1-shear-resistant as well. On the other hand, $C_{5}=\hat{Z}^{(2)}$ cannot be deformed nontrivially preserving the coplanarity of the marked paraxial rectangle. Hence, $C_{5} \in \mathcal{S}_{2} \backslash \mathcal{S}_{1}$.

Configuration $C_{2}$ in Figure 5 is an example of a configuration which is not $k$-shearresistant for any $k \in \mathbb{N}_{0}$ (in fact it is the smallest configuration in the sense of number of points which is not in $\left.\mathcal{S}_{0}\right)$. Indeed, we have that $\left(C_{2}\right)^{(k)}=C_{2}$ for all $k \in \mathbb{N}$, as it consists of a single cell, so that no additional angle-rigidity can follow by taking $k$ large. Note that the property of not belonging to $\mathcal{S}_{k}$ for any $k \in \mathbb{N}_{0}$ does not necessarily imply that the configuration is small. Indeed, Figure 6 below shows that configurations which are not $k$-shear-resistant for any $k \in \mathbb{N}_{0}$ can be arbitrarily large.

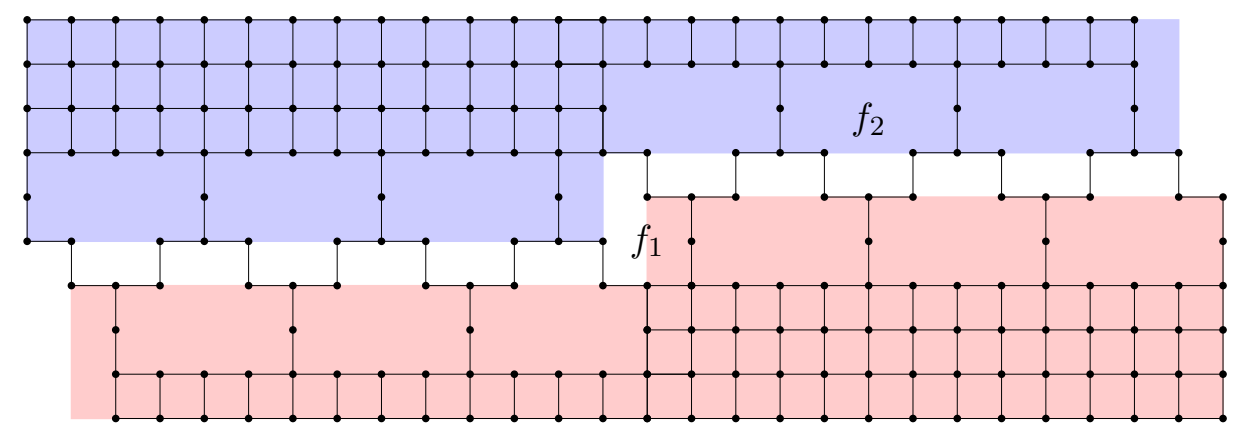

Figure 6. Example of a configuration $C \notin \mathcal{S}_{k}$ for all $k \in \mathbb{N}_{0}$. Indeed, the faces $f_{2}$ are 1 -shear-resistant, but $f_{1}$ is not $k$-shear-resistant for all $k \in \mathbb{N}_{0}$. (The corresponding nontrivial mapping is the one indicated by the colors: leave the points in the red region in $\mathbb{R}^{2} \times\{0\}$ and move the points in the blue region by the vector $(0,1-\cos t, \sin t)$ for any $t>0$ small.) The configuration extends the example provided in Figure 3.

The focus of this section is that of proving that condition (6) indeed characterizes angle-rigidity in $\mathcal{S}_{k}$. More precisely, we have the following.

Proposition 5.2 ( Characterization, shear-resistance). Consider $C \in \mathcal{S}_{k}$ for some $k \in \mathbb{N}_{0}$. Then, $C$ is not angle-rigid iff condition (6) holds. 
Recall that the $\mathcal{S}_{k}$ assumption in Proposition 5.2 cannot be removed. In fact, Figures 3 and 6 give examples of not angle-rigid configurations which do not fulfill (6).

Proof of Proposition 5.2. If condition (6) holds, then $C$ is not angle-rigid by Proposition 4.1. Thus, we only need to show the other implication. We start by pointing out an aspect of Definition 5.1. Given any cycle in the bond structure of a $k$-shear-resistant configuration $C$, and four different points $\boldsymbol{x}_{i}, \boldsymbol{x}_{i^{\prime}}, \boldsymbol{x}_{j}, \boldsymbol{x}_{j^{\prime}}$ with $\left(\boldsymbol{x}_{i}, \boldsymbol{x}_{i^{\prime}}\right),\left(\boldsymbol{x}_{j}, \boldsymbol{x}_{j^{\prime}}\right) \in \boldsymbol{N}(C)$ which form a paraxial rectangle whose interior is contained in the interior of the cycle, then

$$
\boldsymbol{\varphi}\left(\boldsymbol{x}_{i}\right), \boldsymbol{\varphi}\left(\boldsymbol{x}_{i^{\prime}}\right), \boldsymbol{\varphi}\left(\boldsymbol{x}_{j}\right), \boldsymbol{\varphi}\left(\boldsymbol{x}_{j^{\prime}}\right) \text { are coplanar }
$$

for each angle-preserving mapping $\varphi$. In fact, if this were not the case, we would find a cell in the interior of the cycle and a corresponding paraxial rectangle whose images under $\varphi$ are not coplanar. Therefore, this cell would not be $k$-shear-resistant.

As $C$ is not angle-rigid, we find a nontrivial angle-preserving mapping $\varphi$. In particular, the points $\varphi(C) \subset \mathbb{R}^{3}$ are not coplanar as mappings $\varphi$ keeping the points coplanar are necessarily trivial. As $C$ is connected, we can hence find a path $\left(\boldsymbol{x}_{1}, \ldots, \boldsymbol{x}_{m}\right) \subset C$ such that $\left\{\boldsymbol{\varphi}\left(\boldsymbol{x}_{i}\right)\right\}_{i=2}^{m-1}$ are all contained in a line, but $\left\{\boldsymbol{\varphi}\left(\boldsymbol{x}_{i}\right)\right\}_{i=1}^{m}$ are not coplanar. Without restriction we can choose the number $m$ minimal, i.e., for all paths consisting of at most $(m-1)$ points the images under $\varphi$ are coplanar. Moreover, with no loss of generality, by possibly changing coordinates we can assume that $\varphi\left(\boldsymbol{x}_{i}\right)=\boldsymbol{x}_{i}$ for all $i=1 \ldots, m-1,\left\{\boldsymbol{x}_{i}\right\}_{i=2}^{m-1}$ are contained in the axis $\mathbb{Z} \times\{0\}, \boldsymbol{x}_{1}=(0,1)$, and

$$
\boldsymbol{x}_{m} \in\{(m-3,1),(m-3,-1)\} .
$$

We also note that $\boldsymbol{\varphi}\left(\boldsymbol{x}_{m}\right) \in\{m-3\} \times \mathbb{R}^{2}$. The minimality of $m$ implies that all points $\left\{\boldsymbol{x}_{i}\right\}_{i=3}^{m-2}$ do not have neighbors off the axis $\mathbb{Z} \times\{0\}$. Indeed, such a neighbor, the axis $\mathbb{Z} \times\{0\}$, and one of the points $\boldsymbol{x}_{1}$ or $\boldsymbol{x}_{m}$ would not remain coplanar by the mapping $\boldsymbol{\varphi}$ and one could hence find a shorter noncoplanar path.

We now suppose by contradiction that condition (6) does not hold, i.e., the configuration $\widetilde{C}$ obtained by removing all points of $C$ belonging to $\mathbb{Z} \times\{0\}$ and having at most one neighbor off $\mathbb{Z} \times\{0\}$ is still connected. Therefore, there exists a path in $\widetilde{C}$, denoted by $P$, connecting $\boldsymbol{x}_{1}$ and $\boldsymbol{x}_{m}$. Note that the points $\left\{\boldsymbol{x}_{i}\right\}_{i=3}^{m-2}$ are not contained in $\widetilde{C}$ as they do not have neighbors off axis. Consequently, there exists a cycle $S$ in $C$, being a subset of the points $P \cup\left\{\boldsymbol{x}_{i}\right\}_{i=2}^{m-1}$. We now argue that it is not restrictive to assume that $\boldsymbol{x}_{1}$ and $\boldsymbol{x}_{m}$ are contained in the cycle $S$ or in the interior of the cycle $S$. In fact, at least one of the points $\boldsymbol{x}_{1}=(0,1)$ and $(0,-1)$ is contained in the cycle $S$ or in the interior of the cycle $S$. If only $(0,-1)$ satisfies this property, this necessarily implies that $(0,0) \in P$ and therefore $(0,-1) \in C$ by $(6)$. Thus, we can simply replace $\boldsymbol{x}_{1}$ by $(0,-1)$ in the path $P$ observing that $\varphi(0,-1)=(0,-1)$ by property (4). In a similar fashion, by possibly reflecting $\boldsymbol{x}_{m}$ along $\mathbb{R} \times\{0\}$ we can suppose that $\boldsymbol{x}_{m}$ is contained in the cycle $S$ or in the interior of the cycle $S$.

We now distinguish two cases: (a) $\boldsymbol{x}_{m}=(m-3,1)$ and (b) $\boldsymbol{x}_{m}=(m-3,-1)$, cf. 10. 
In case (a), the points $\boldsymbol{x}_{1}, \boldsymbol{x}_{2}, \boldsymbol{x}_{m-1}, \boldsymbol{x}_{m}$ form a paraxial rectangle whose interior is contained in the interior of $S$. (Recall that $\boldsymbol{x}_{1}=(0,1)$ and $\boldsymbol{x}_{2}=(0,0)$.) Then, (9) implies that $\boldsymbol{\varphi}\left(\boldsymbol{x}_{1}\right), \boldsymbol{\varphi}\left(\boldsymbol{x}_{2}\right), \boldsymbol{\varphi}\left(\boldsymbol{x}_{m-1}\right), \boldsymbol{\varphi}\left(\boldsymbol{x}_{m}\right)$ are coplanar. This, however, contradicts our choice of the path $\left(\boldsymbol{x}_{1}, \ldots, \boldsymbol{x}_{m}\right)$.

Consider now case (b). We first observe that the path $P$ in $\widetilde{C}$ connecting $\boldsymbol{x}_{1}$ and $\boldsymbol{x}_{m}$ needs to cross the axis $\mathbb{Z} \times\{0\}$, i.e., we find $\overline{\boldsymbol{x}} \in(\mathbb{Z} \times\{0\}) \cap P$. We observe that it is not restrictive to suppose that also

$$
\overline{\boldsymbol{x}}+(0,1) \in P, \quad \overline{\boldsymbol{x}}-(0,1) \in P .
$$

In fact, consider the smallest subpath $\left(\hat{\boldsymbol{x}}_{1}, \ldots, \hat{\boldsymbol{x}}_{l}\right)$ of $P \subset \widetilde{C}$ containing $\overline{\boldsymbol{x}}$ such that $\hat{\boldsymbol{x}}_{1} \in \mathbb{Z} \times\{-1\},\left\{\hat{\boldsymbol{x}}_{i}\right\}_{i=2}^{l-1} \subset \mathbb{Z} \times\{0\}$, and $\hat{\boldsymbol{x}}_{l} \in \mathbb{Z} \times\{1\}$. Then, by the definition of $\widetilde{C}$ we have that $\left\{\hat{\boldsymbol{x}}_{i}+(0,1)\right\}_{i=2}^{l-1} \subset \widetilde{C}$ and $\left\{\hat{\boldsymbol{x}}_{i}-(0,1)\right\}_{i=2}^{l-1} \subset \widetilde{C}$. This means that we can connect $\hat{\boldsymbol{x}}_{1}$ and $\hat{\boldsymbol{x}}_{l}$ with a path in $\widetilde{C}$ which contains only one point of the axis $\mathbb{Z} \times\{0\}$. By replacing the part $\left(\hat{\boldsymbol{x}}_{1}, \ldots, \hat{\boldsymbol{x}}_{l}\right)$ of $P$ by this new path, we obtain the desired property (11).

Define $\overline{\boldsymbol{x}}^{\prime}=\overline{\boldsymbol{x}}-(0,1)$ and $\overline{\boldsymbol{x}}^{\prime \prime}=\overline{\boldsymbol{x}}+(0,1)$ for brevity, and recall that $\overline{\boldsymbol{x}}^{\prime}, \overline{\boldsymbol{x}}^{\prime \prime} \in P$ by (11). If the path $P$ crosses the axis $\mathbb{Z} \times\{0\}$ several times, we choose $\overline{\boldsymbol{x}} \in(\mathbb{Z} \times\{0\}) \cap P$ to be the point closest to $\boldsymbol{x}_{2}$. Then, we observe that $\boldsymbol{x}_{1}, \boldsymbol{x}_{2}, \overline{\boldsymbol{x}}, \overline{\boldsymbol{x}}^{\prime \prime}$ and $\boldsymbol{x}_{m-1}, \boldsymbol{x}_{m}, \overline{\boldsymbol{x}}, \overline{\boldsymbol{x}}^{\prime}$ form paraxial rectangles whose interior is contained in the interior of the cycle $S$. In view of (4), we get that the points $\varphi(\bar{x}), \varphi\left(\overline{\boldsymbol{x}}^{\prime}\right), \boldsymbol{\varphi}\left(\overline{\boldsymbol{x}}^{\prime \prime}\right)$ lie on a line. Moreover, as $\boldsymbol{x}_{1}, \boldsymbol{x}_{2}, \overline{\boldsymbol{x}}, \overline{\boldsymbol{x}}^{\prime \prime}$ form a paraxial rectangle whose interior is contained in the interior of the cycle $S$, we find that $\varphi\left(\boldsymbol{x}_{1}\right), \boldsymbol{\varphi}\left(\boldsymbol{x}_{2}\right), \boldsymbol{\varphi}(\overline{\boldsymbol{x}}), \boldsymbol{\varphi}\left(\overline{\boldsymbol{x}}^{\prime \prime}\right)$ are also coplanar by $(9)$. In a similar fashion, $\boldsymbol{x}_{m-1}, \boldsymbol{x}_{m}, \overline{\boldsymbol{x}}, \overline{\boldsymbol{x}}^{\prime}$ form a paraxial rectangle contained in the interior of cycle $S$, and thus $\boldsymbol{\varphi}\left(\boldsymbol{x}_{m-1}\right), \boldsymbol{\varphi}\left(\boldsymbol{x}_{m}\right), \boldsymbol{\varphi}(\overline{\boldsymbol{x}}), \boldsymbol{\varphi}\left(\overline{\boldsymbol{x}}^{\prime}\right)$ are coplanar. This entails that also $\left\{\boldsymbol{\varphi}\left(\boldsymbol{x}_{i}\right)\right\}_{i=1}^{m}$ are coplanar which clearly contradicts our choice of the path $\left(\boldsymbol{x}_{1}, \ldots, \boldsymbol{x}_{m}\right)$. This concludes the proof.

We close this discussion by summarizing the results of Sections 45 in the following statement.

Corollary 5.3 (Necessary and sufficient condition). For $C \in \mathcal{F} \cup \mathcal{S}_{k}$ for some $k \in \mathbb{N}_{0}$ we have that $C$ is not angle-rigid iff condition (6) holds.

Note that $\mathcal{F} \cup \mathcal{S}_{k}$ does not exhaust all possible configurations. More precisely, the situation is depicted in Figure 7 below: as $k$ grows, $\mathcal{S}_{k}$ covers all angle-rigid configurations and configurations in $\mathcal{S}_{k}$ not being angle-rigid but belonging to $\mathcal{F}$ (Proposition 5.2). There exist not angle-rigid configurations which are not in $\mathcal{F}$ (see Figure 3), as well as configurations in $\mathcal{F}$ (hence not angle-rigid by definition) that are not in $\mathcal{S}_{k}$ for any $k \in \mathbb{N}_{0}$ (see left-most configuration in Figure 5).

\section{Characterization of 0-Shear-Resistant Cells}

Within the class $\mathcal{F} \cup \mathcal{S}_{k}$, Corollary 5.3 reduces the problem of assessing angle-rigidity to that of verifying condition (6). Therefore, we are interested in checking if a configuration $C \in \mathbb{Z}^{2 n}$ lies in $\mathcal{F} \cup \mathcal{S}_{k}$. As $C \in \mathcal{F}$ is equivalent to condition (6) by Proposition 4.3. one is hence left to assess if there exists $k \in \mathbb{N}_{0}$ such that $C \in \mathcal{S}_{k}$. 


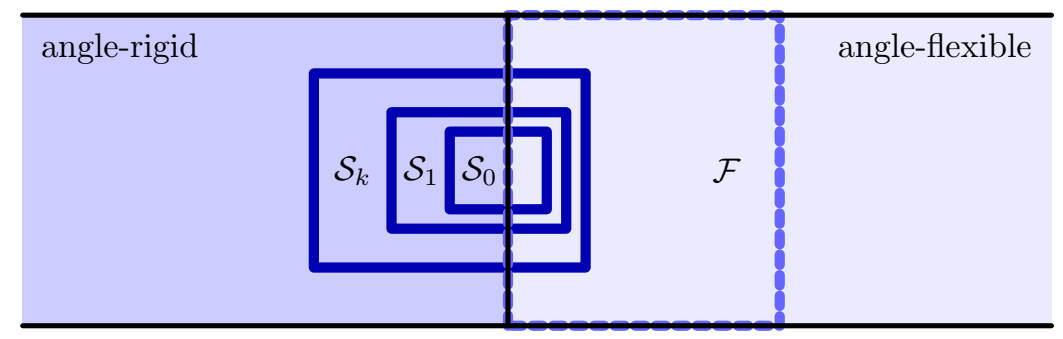

Figure 7. The classes $\mathcal{F}$ (dashed) and $\mathcal{S}_{k}$ (solid) in relation with angle-rigidity.

To check if $C \in \mathcal{S}_{k}$ can still be very demanding from an computational viewpoint. Consider again the example in Figure 6, where the configuration does not belong to $\mathcal{S}_{k}$ for any $k \in \mathbb{N}_{0}$ (and indeed is angle-flexible). Adding a bonded point on the top of the rightmost line makes the rightmost cell with face $f_{2}$ rigid. This augmented configuration turns out to belong to $\mathcal{S}_{k}$ for some $k \in \mathbb{N}$.

This in particular entails that, in general, belonging to class $\mathcal{S}_{k}$ is a global property, and checking it requires the analysis of the whole configuration.

The focus of this section is on the class $\mathcal{S}_{0}$. This is special, for we are able to present complete characterizations based on localized arguments.

We start by introducing some notation. Let a cell $Z \in \mathcal{Z}(C)$ be given. Referring to the discussion at the beginning of Section 5 , we suppose that $Z$ does not contain acyclic bonds. Therefore, the boundary of its face $f(Z)$ is a (simple) polygon. We denote the points of the cell by $\left\{\boldsymbol{p}^{1}, \ldots, \boldsymbol{p}^{m}\right\}$ with $\left|\boldsymbol{p}^{i}-\boldsymbol{p}^{i+1}\right|=1$ for $i=1, \ldots, m$, where we understand the indices modulo $m$, if necessary. Note that the points are not necessarily pairwise different. We say that a cell is simple if $\left|\boldsymbol{p}^{i}-\boldsymbol{p}^{j}\right|>1$ for all $i, j$ with $|i-j| \geq 2$. We refer to Figure 8 for examples of nonsimple cells. In case that $Z$ is not simple, the bond structure of $Z$ is delimited by a minimal (in the sense of set inclusion) polygon of points of $Z$, which we call the outer polygon. Correspondingly, the points of $Z$ in the outer polygon form a cell, which we call the outer cell, denoted by $Z^{\text {out }}$. Up to a negligible set, the set $f\left(Z^{\text {out }}\right) \backslash f(Z)$ is the union of faces corresponding to other cells of the bond structure. These cells are called the inner cells corresponding to $Z$ and their collection is indicated by $\mathcal{I}_{Z}$. For simple cells $Z$, we have $Z=Z^{\text {out }}$ and $\mathcal{I}_{Z}=\emptyset$. Note that $Z^{\text {out }}$ and $\mathcal{I}_{Z}$ are simple cells. In fact, if an inner cell were not simple, it could be decomposed into two smaller cells. If the outer cell were not simple, there would exist a polygon with less points of $Z$ delimiting $Z$.

We denote the vertices of the polygon $\partial f(Z)$ by $\left\{\boldsymbol{v}^{1}, \ldots, \boldsymbol{v}^{n}\right\}$ for some $n \in 2 \mathbb{N}$, ordered counterclockwise. (Here, $n$ is unrelated to the number of points in a configuration.) We understand the indices modulo $n$, if necessary. Clearly, we have $\left\{\boldsymbol{v}^{1}, \ldots, \boldsymbol{v}^{n}\right\} \subset$ $\left\{\boldsymbol{p}^{1}, \ldots, \boldsymbol{p}^{m}\right\}$, where in general the inclusion is strict. We decompose the set of the vertices of the polygon into the right- and left-set

$$
I_{1,+}(Z)=\left\{\boldsymbol{v}^{i}: \boldsymbol{v}^{i+1}-\boldsymbol{v}^{i} \in \mathbb{N}(1,0)\right\}, \quad I_{1,-}(Z)=\left\{\boldsymbol{v}^{i}: \boldsymbol{v}^{i+1}-\boldsymbol{v}^{i} \in \mathbb{N}(-1,0)\right\}
$$



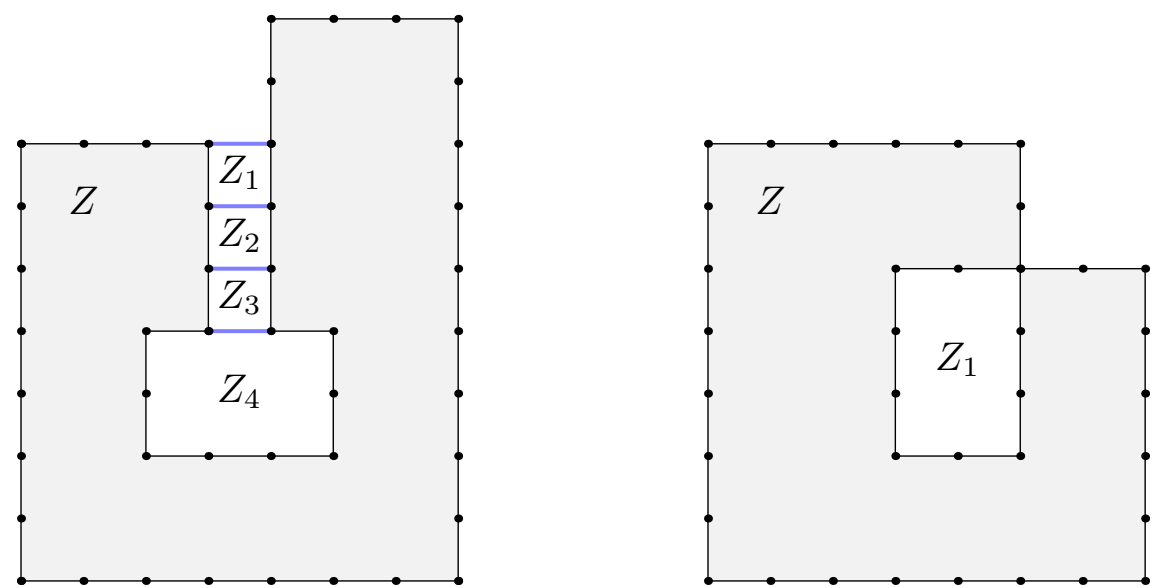

Figure 8. Examples of nonsimple cells. Here, $f(Z)$ is shaded and $Z_{i} \in$ $\mathcal{I}_{Z}$ are inner cells.

and the up- and down-set

$$
I_{2,+}(Z)=\left\{\boldsymbol{v}^{i}: \boldsymbol{v}^{i+1}-\boldsymbol{v}^{i} \in \mathbb{N}(0,1)\right\}, \quad I_{2,-}(Z)=\left\{\boldsymbol{v}^{i}: \boldsymbol{v}^{i+1}-\boldsymbol{v}^{i} \in \mathbb{N}(0,-1)\right\} .
$$

Note that we necessarily have that $\# I_{j, \pm}(Z) \geq 1$ for $j=1,2$.

With the aim of proving a characterization of 0 -shear-resistant cells, we address the case of simple cells first.

Proposition 6.1 (Characterization of 0-shear-resistance, simple cells). A simple cell $Z$ is 0 -shear-resistant iff $\min \left\{\# I_{1,+}(Z), \# I_{1,-}(Z)\right\}=\min \left\{\# I_{2,+}(Z)\right.$, \# $\left.I_{2,-}(Z)\right\}=1$ iff the configuration consisting of $Z$ is angle-rigid.

For the proof of Proposition 6.1, we will make use of the elementary geometrical construction described in the following lemma.

Lemma 6.2 (Geometric construction). Let $\boldsymbol{y}^{1}, \boldsymbol{y}^{2}, \boldsymbol{y}^{3}, \boldsymbol{y}^{4} \in\{0\} \times \mathbb{R} \times\{0\}$ be given. Suppose that the second components of these vectors satisfy
(a) $y_{2}^{1}<y_{2}^{4} \leq y_{2}^{2}<y_{2}^{3}$
or
(b) $y_{2}^{1} \leq y_{2}^{3}<y_{2}^{4} \leq y_{2}^{2}$.

Then, for $t>0$ small, there exist $\tilde{\boldsymbol{y}}_{t}^{3}, \tilde{\boldsymbol{y}}_{t}^{4} \in\{0\} \times \mathbb{R}^{2}$ with $\tilde{\boldsymbol{y}}_{t}^{3} \neq \boldsymbol{y}^{3}, \tilde{\boldsymbol{y}}_{t}^{4} \neq \boldsymbol{y}^{4}, \tilde{\boldsymbol{y}}_{t}^{3} \rightarrow \boldsymbol{y}^{3}$, and $\tilde{\boldsymbol{y}}_{t}^{4} \rightarrow \boldsymbol{y}^{4}$ as $t \rightarrow 0$ such that

$$
\left|\boldsymbol{y}^{1}-\tilde{\boldsymbol{y}}_{t}^{4}\right|=\left|\boldsymbol{y}^{1}-\boldsymbol{y}^{4}\right|, \quad\left|\boldsymbol{y}^{2}-\tilde{\boldsymbol{y}}_{t}^{3}\right|=\left|\boldsymbol{y}^{2}-\boldsymbol{y}^{3}\right|, \quad \text { and } \quad\left|\tilde{\boldsymbol{y}}_{t}^{3}-\tilde{\boldsymbol{y}}_{t}^{4}\right|=\left|\boldsymbol{y}^{3}-\boldsymbol{y}^{4}\right| .
$$

Proof. For simplicity, we only prove case (a). Case (b) follows along similar lines with a different notational realization. Given $t>0$, let $\tilde{\boldsymbol{y}}_{t}^{4}=\boldsymbol{y}^{4}+(0, s(t), t)$ with $t \mapsto s(t)<0$ such that $\left|\boldsymbol{y}^{1}-\tilde{\boldsymbol{y}}_{t}^{4}\right|=\left|\boldsymbol{y}^{1}-\boldsymbol{y}^{4}\right|$ holds (note that necessarily $s(t)=\mathrm{O}\left(t^{2}\right)$ ). As a next step, we let $\tilde{\boldsymbol{y}}_{t}^{3}=\left(0, x_{2}^{t}, x_{3}^{t}\right)$ where $x_{3}^{t}$ solves

$$
y_{2}^{2}+\sqrt{\left|\boldsymbol{y}^{2}-\boldsymbol{y}^{3}\right|^{2}-\left(x_{3}^{t}\right)^{2}}=y_{2}^{4}+s(t)+\sqrt{\left|\boldsymbol{y}^{3}-\boldsymbol{y}^{4}\right|^{2}-\left(x_{3}^{t}-t\right)^{2}}
$$


and $x_{2}^{t}$ is defined by

$$
x_{2}^{t}=y_{2}^{2}+\sqrt{\left|\boldsymbol{y}^{2}-\boldsymbol{y}^{3}\right|^{2}-\left(x_{3}^{t}\right)^{2}} .
$$

Note that the above equations imply that $\left|\boldsymbol{y}^{2}-\tilde{\boldsymbol{y}}_{t}^{3}\right|=\left|\boldsymbol{y}^{2}-\boldsymbol{y}^{3}\right|$ and $\left|\tilde{\boldsymbol{y}}_{t}^{3}-\tilde{\boldsymbol{y}}_{t}^{4}\right|=\left|\boldsymbol{y}^{3}-\boldsymbol{y}^{4}\right|$. By checking that $x_{3}^{t}=\mathrm{O}(t)$ as $t \rightarrow 0$ we hence obtain the assertion.

One is therefore left to prove that equation (13) has at least one solution $x_{3}^{t}$ with $x_{3}^{t}=\mathrm{O}(t)$. An elementary albeit tedious computation allows to rewrite $(13)$ as $2 t x_{3}^{t}=$ $g_{t}\left(x_{3}^{t}\right)$, where

$$
g_{t}\left(x_{3}^{t}\right):=t^{2}+s^{2}+2(b+s)(b-a)+2(a-b-s) \sqrt{b^{2}-\left(x_{3}^{t}\right)^{2}},
$$

where we have used the shorthand notation $a:=\left|\boldsymbol{y}^{3}-\boldsymbol{y}^{4}\right|, b=\left|\boldsymbol{y}^{2}-\boldsymbol{y}^{3}\right|$, and $s=s(t)$. Recall that $a \geq b$ by assumption and that $s<0$.

If $a>b$, we obtain $g_{t}(0)>0$ and $g_{t}(b)<0$ for $t$ (and thus $s$ ) sufficiently small. Therefore, by the Intermediate Value Theorem applied to the continuous function $x \mapsto$ $-2 t x+g_{t}(x)$ we find $x_{3}^{t} \in(0, b)$ such that (14) holds. By observing that $g_{t}$ is maximized at $x=0$, since $\max g_{t}=g_{t}(0)=\mathrm{O}\left(t^{2}+s^{2}+s\right)=\mathrm{O}\left(t^{2}\right)$, we also get $x_{3}^{t}=\mathrm{O}(t)$.

If $a=b$, we find $g_{t}(0)>0$ and $2 b t>g_{t}(b)=t^{2}+s^{2}$ for $t$ sufficiently small. Hence, also in this case (14) has a solution $x_{3}^{t}$ with $x_{3}^{t}=\mathrm{O}(t)$.

Having prepared the approximation tool of Lemma 6.2, we can now proceed to the proof of Proposition 6.1.

Proof of Proposition 6.1. Suppose that $Z$ is simple. Assume first that

$$
\min \left\{\# I_{1,+}, \# I_{1,-}\right\}=\min \left\{\# I_{2,+}, \# I_{2,-}\right\}=1 .
$$

(Here and in the sequel, we drop ' $(Z)^{\prime}$ ' from the notation for simplicity.) We want to prove that $Z$ is angle-rigid. After rotation, reflection, and relabeling of the vertices, it is not restrictive to suppose that $\boldsymbol{v}^{1} \in I_{1,+}, \boldsymbol{v}^{2} \in I_{2,+}, \boldsymbol{v}^{1+2 i} \in I_{1,-}$ for $i=1, \ldots, n / 2-1$, and $\boldsymbol{v}^{2+2 i} \in I_{2,-}$ for $i=1, \ldots, n / 2-1$, see Figure 9 .

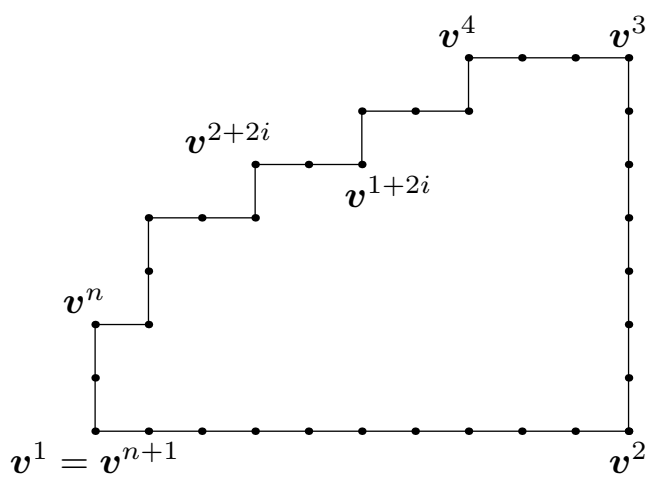

Figure 9. The points $\boldsymbol{v}^{i}$.

Let $\varphi$ be an angle-preserving mapping on $Z$. We may assume without restriction that $\boldsymbol{\varphi}\left(\boldsymbol{v}^{i}\right)=\boldsymbol{v}^{i}$ for $i=1,2,3$. Recalling the identification $\boldsymbol{v}^{n+1}=\boldsymbol{v}^{1}$ this particularly 
implies that $\left|\boldsymbol{\varphi}\left(\boldsymbol{v}^{3}\right)-\boldsymbol{\varphi}\left(\boldsymbol{v}^{n+1}\right)\right|=\left|\boldsymbol{v}^{3}-\boldsymbol{v}^{n+1}\right|$. As $\boldsymbol{v}^{1+2 i} \in I_{1,-}$ for $i=1, \ldots, n / 2-1$ and $\boldsymbol{v}^{2+2 i} \in I_{2,-}$ for $i=1, \ldots, n / 2-1$, this is only possible if the points $\left\{\boldsymbol{\varphi}\left(\boldsymbol{v}^{i}\right)\right\}_{i=3}^{n+1}$ are coplanar. Indeed, for any $4 \leq i \leq n-1$, a rotation of the points $\boldsymbol{v}^{i}, \ldots, \boldsymbol{v}^{n+1}$ around the axis containing the points $\boldsymbol{v}^{i}$ and $\boldsymbol{v}^{i+1}$ decreases the distance between $\boldsymbol{v}^{3}$ and $\boldsymbol{v}^{n+1}$. By using (4) for $\left(\boldsymbol{v}^{n}, \boldsymbol{v}^{1}, \boldsymbol{v}^{2}\right)$ and $\left(\boldsymbol{v}^{2}, \boldsymbol{v}^{3}, \boldsymbol{v}^{4}\right)$ this yields that $\left\{\boldsymbol{\varphi}\left(\boldsymbol{v}^{i}\right)\right\}_{i=3}^{n+1}$ are contained in $\mathbb{Z}^{2} \times\{0\}$, and therefore $\varphi$ is the identity. This proves that $Z$ is angle-rigid.

Next, if $Z$ is assumed to be angle-rigid, the validity of Condition (i) of Definition 5.1 clearly follows, i.e., $Z$ is 0 -shear resistant.

Eventually, we assume that $Z$ is 0 -shear resistant and show that $\min \left\{\# I_{1,+}, \# I_{1,-}\right\}=$ $\min \left\{\# I_{2,+}, \# I_{2,-}\right\}=1$. We show the contrapositive: suppose that $\min \left\{\# I_{2,+}, \# I_{2,-}\right\} \geq$ 2 and we prove that $Z$ is not 0 -shear-resistant (the case $\min \left\{\# I_{1,+}, \# I_{1,-}\right\} \geq 2$ is analogous). Up to a cyclic relabeling of the vertices, we can reduce the problem to the following two different cases: we have $\left\{\boldsymbol{x}^{1}, \ldots, \boldsymbol{x}^{4}\right\}$ with $\boldsymbol{x}^{i}=\boldsymbol{v}^{m(i)}$ for $i=1, \ldots, 4$, where $m:\{1, \ldots, 4\} \rightarrow\{1, \ldots, n\}$ is strictly increasing, such that one of the following two cases hold

(a) $\boldsymbol{x}^{1}, \boldsymbol{x}^{2} \in I_{2,+}$ and $\boldsymbol{x}^{3}, \boldsymbol{x}^{4} \in I_{2,-}$ with $x_{2}^{1}<x_{2}^{2}<x_{2}^{3}$ and $x_{2}^{1}<x_{2}^{4}<x_{2}^{3}$,

(b) $\boldsymbol{x}^{1}, \boldsymbol{x}^{3} \in I_{2,+}$ and $\boldsymbol{x}^{2}, \boldsymbol{x}^{4} \in I_{2,-}$ with $x_{2}^{1} \leq x_{2}^{3}<x_{2}^{2}$ and $x_{2}^{3}<x_{2}^{4}$,

see Figure 10.

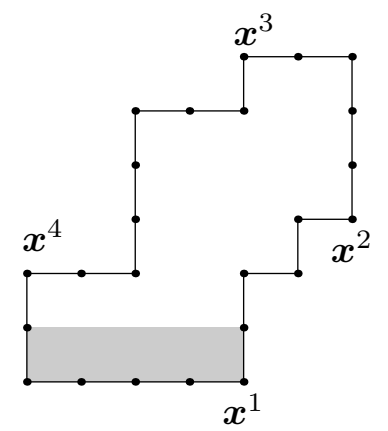

(a)

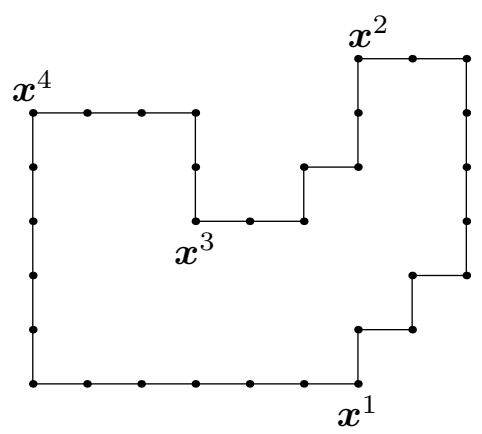

(b)

Figure 10. The two cases from (15)-16). The shaded region is the paraxial rectangle mentioned in the proof.

We treat case (a) first. After reflection of the cell along the $x_{2}$-axis and interchanging the labels for $\boldsymbol{x}^{2}$ and $\boldsymbol{x}^{4}$, we may suppose that $x_{2}^{4} \leq x_{2}^{2}$. We denote the orthogonal projection of $\boldsymbol{x}^{i}$ onto $\{0\} \times \mathbb{R} \times\{0\}$ by $\boldsymbol{y}^{i}$ and note that $y_{2}^{1}<y_{2}^{4} \leq y_{2}^{2}<y_{2}^{3}$. We apply Lemma 6.2(a) and obtain two points $\tilde{\boldsymbol{y}}_{t}^{3}, \tilde{\boldsymbol{y}}_{t}^{4} \in\{0\} \times \mathbb{R}^{2}$ for $t>0$ small such that (12) holds. By $R_{t}^{23}$ we denote the rotation in $\mathbb{R}^{3}$ with axis parallel to the $x_{1}$-axis which leaves $\boldsymbol{x}^{2}$ unchanged and moves $\boldsymbol{x}^{3}$ to $\boldsymbol{x}^{3}+\tilde{\boldsymbol{y}}_{t}^{3}-\boldsymbol{y}_{t}^{3}$. By $R_{t}^{34}$ we denote the rotation in $\mathbb{R}^{3}$ with axis parallel to the $x_{1}$-axis which moves $\boldsymbol{x}^{3}$ to $\boldsymbol{x}^{3}+\tilde{\boldsymbol{y}}_{t}^{3}-\boldsymbol{y}^{3}$ and moves $\boldsymbol{x}^{4}$ to $\boldsymbol{x}^{4}+\tilde{\boldsymbol{y}}_{t}^{4}-\boldsymbol{y}^{4}$. Finally, by $R_{t}^{14}$ we denote the rotation in $\mathbb{R}^{3}$ with axis parallel to the $x_{1}$-axis which leaves $\boldsymbol{x}^{1}$ unchanged and moves $\boldsymbol{x}^{4}$ to $\boldsymbol{x}^{4}+\tilde{\boldsymbol{y}}_{t}^{4}-\boldsymbol{y}^{4}$. Note that these 
isometries exist due to $(12)$. We now define $\boldsymbol{\varphi}$ on the vertices $\left\{\boldsymbol{v}^{1}, \ldots, \boldsymbol{v}^{n}\right\}$ by

$$
\boldsymbol{\varphi}\left(\boldsymbol{v}^{i}\right)= \begin{cases}\boldsymbol{v}^{i} & \text { if } m(1) \leq i \leq m(2), \\ R_{t}^{23}\left(\boldsymbol{v}^{i}\right) & \text { if } m(2) \leq i \leq m(3), \\ R_{t}^{34}\left(\boldsymbol{v}^{i}\right) & \text { if } m(3) \leq i \leq m(4), \\ R_{t}^{14}\left(\boldsymbol{v}^{i}\right) & \text { if } m(4) \leq i \leq m(1)\end{cases}
$$

(Note that the definition is consistent for $i=m(j)$ for $j=1, \ldots, 4$.) This mapping can be naturally extended to the points $\left\{\boldsymbol{p}^{1}, \ldots, \boldsymbol{p}^{m}\right\}$ of the cell $Z$ : given a point $\boldsymbol{p}^{j} \notin\left\{\boldsymbol{v}^{1}, \ldots, \boldsymbol{v}^{n}\right\}$ lying on the segment between $\boldsymbol{v}^{i}$ and $\boldsymbol{v}^{i+1}$, we set $\boldsymbol{\varphi}\left(\boldsymbol{p}^{j}\right)=R_{t}\left(\boldsymbol{p}^{j}\right)$, where $R_{t}$ denotes the isometry under which both $\boldsymbol{v}^{i}$ and $\boldsymbol{v}^{i+1}$ are moved (i.e., the identity or $\left.R_{t}^{23}, R_{t}^{34}, R_{t}^{14}\right)$. By construction we can check that $\varphi$ is angle-preserving. Indeed, we observe that all bonds of $Z$ only join consecutive points $\boldsymbol{p}^{i}$ and $\boldsymbol{p}^{i+1}, i=1, \ldots, m$, since $Z$ is simple. Then, the fact that the mappings $R_{t}^{23}, R_{t}^{34}$, and $R_{t}^{14}$ are isometries implies (3). In order to check (4), we additionally use that the isometries are actually rotations about axes parallel to the $x_{1}$-axis and all bonds of $Z$ are of course either parallel or orthogonal to $x_{1}$. Moreover, since $R_{t}^{14}$ is not the identity, we find that the condition in Definition 5.1(i) is violated for at least one paraxial rectangle (shaded region in Figure 10 left).

The case (b) is very similar and we only indicate the necessary adaptations. After reflection of the cell along the $x_{2}$-axis, we may suppose that $x_{2}^{4} \leq x_{2}^{2}$. As before, we indicate by $\boldsymbol{y}^{i}$ the orthogonal projection of $\boldsymbol{x}^{i}$ onto $\{0\} \times \mathbb{R} \times\{0\}$ and note that $y_{2}^{1} \leq y_{2}^{3}<y_{2}^{4} \leq y_{2}^{2}$. We now apply Lemma 6.2(b) and argue similarly as before to define $\varphi$.

We now proceed with the characterization of 0 -shear-resistance for general cells, i.e., for possibly not simple cells. To this end, recall the notions introduced at the beginning of Section 6 .

Proposition 6.3 (Characterization of 0 -shear-resistance). A cell $Z \in \mathcal{Z}(C)$ is 0 -shearresistant if and only if

$$
\begin{aligned}
& Z \notin \mathcal{F} \text { and } \\
& \min \left\{\# I_{1,+}\left(Z^{\prime}\right), \# I_{1,-}\left(Z^{\prime}\right)\right\}=\min \left\{\# I_{2,+}\left(Z^{\prime}\right), \# I_{2,-}\left(Z^{\prime}\right)\right\}=1 \quad \forall Z^{\prime} \in\left\{Z^{\text {out }}\right\} \cup \mathcal{I}_{Z} .
\end{aligned}
$$

Proof. We start with a preliminary observation. Consider a cell in $Z^{\prime} \in\left\{Z^{\text {out }}\right\} \cup \mathcal{I}_{Z}$. Then, each cell $\tilde{Z} \in\left\{Z^{\text {out }}\right\} \cup \mathcal{I}_{Z}$ with $\tilde{Z} \neq Z^{\prime}$ and $Z^{\prime} \cap \tilde{Z} \neq \emptyset$ shares at most one bond with $Z^{\prime}$. Consequently, for each connected component $\widehat{C}$ of $Z \backslash Z^{\prime}$ there is at least one and at most two points in $Z^{\prime}$ connected by a bond in $Z$ to $\widehat{C}$. (If there are two points, these stay at distance 1.) This directly comes from the definition of $\mathcal{I}_{Z}$ and $Z^{\text {out }}$. In fact, $f(Z)$ and $f\left(Z^{\prime}\right)$ for $Z^{\prime} \in \mathcal{I}_{Z}$ corresponds to a partition of $f\left(Z^{\text {out }}\right)$, and all segments in the bond structure which are not contained in $\partial f(Z)$ have length 1 , see Figure 8.

We now start with the actual proof. Assume first that $Z \notin \mathcal{F}$ and

$$
\min \left\{\# I_{1,+}\left(Z^{\prime}\right), \# I_{1,-}\left(Z^{\prime}\right)\right\}=\min \left\{\# I_{2,+}\left(Z^{\prime}\right), \# I_{2,-}\left(Z^{\prime}\right)\right\}=1 \quad \forall Z^{\prime} \in\left\{Z^{\text {out }}\right\} \cup \mathcal{I}_{Z} \text {. }
$$


Our goal is to show that $Z$ is 0 -shear-resistant. To this end, let $\varphi$ be an angle-preserving mapping of $Z$. As each cell in $\left\{Z^{\text {out }}\right\} \cup \mathcal{I}_{Z}$ is simple, Proposition 6.1 yields that the points of each cell in $\left\{Z^{\text {out }}\right\} \cup \mathcal{I}_{Z}$ remain coplanar under $\varphi$. Moreover, for each $Z^{\prime} \in \mathcal{I}_{Z}$ and each $\boldsymbol{x} \in Z^{\prime}$, we find a path $\left(\boldsymbol{q}^{1}, \ldots, \boldsymbol{q}^{l}\right)$ in $Z$ connecting $\boldsymbol{x}$ with a point $\boldsymbol{y} \in Z^{\text {out }}$ such that, whenever $\boldsymbol{q}^{i-1} \in \tilde{Z}^{\prime} \backslash \tilde{Z}^{\prime \prime}, \boldsymbol{q}^{i} \in \tilde{Z}^{\prime} \cap \tilde{Z}^{\prime \prime}$, and $\boldsymbol{q}^{i+1} \in \tilde{Z}^{\prime \prime} \backslash \tilde{Z}^{\prime}$ for two cells $\tilde{Z}^{\prime}, \tilde{Z}^{\prime \prime} \in\left\{Z^{\text {out }}\right\} \cup \mathcal{I}_{Z}$, then $\boldsymbol{q}^{i+1}-\boldsymbol{q}^{i}=\boldsymbol{q}^{i}-\boldsymbol{q}^{i-1}$. In fact, otherwise we would necessarily find an axis such that (6) holds. Here, we use the preliminary observation that each cell $\tilde{Z}^{\prime}$ contains at most two points which are bonded to the connected component $\widehat{C}$ of $Z \backslash \tilde{Z}^{\prime}$ with $Z^{\text {out }} \backslash \tilde{Z}^{\prime} \subset \widehat{C}$, where, if two points exist, they have distance 1 and thus lie on one axis. This property, however, contradicts $Z \notin \mathcal{F}$ and Proposition 4.3. This can be visualized in Figure 8, left. Take $\tilde{Z}^{\prime}=Z_{3}, \tilde{Z}^{\prime \prime}=Z_{4}$ and let $\boldsymbol{q}^{i}$ be any of the two points in $Z_{3} \cap Z_{4}$. Then, $\boldsymbol{q}^{i+1}-\boldsymbol{q}^{i} \neq \boldsymbol{q}^{i}-\boldsymbol{q}^{i-1}$. Indeed, (6) holds for the axis given by $Z_{3} \cap Z_{4}$, and the configuration turns out to be a folding around this axis.

Now consider some $\boldsymbol{q}^{i} \in \tilde{Z}^{\prime} \cap \tilde{Z}^{\prime \prime}$ as above. Since $\boldsymbol{q}^{i+1}-\boldsymbol{q}^{i}=\boldsymbol{q}^{i}-\boldsymbol{q}^{i-1}$, (4) and the fact that the points of each of the two cells $\tilde{Z}^{\prime}$ and $\tilde{Z}^{\prime \prime}$ remain coplanar under $\varphi$, imply that the points in $\tilde{Z}^{\prime} \cup \tilde{Z}^{\prime \prime}$ remain coplanar under $\varphi$. A successive application of this argument yields that the points in $Z^{\text {out }} \cup Z^{\prime}$ remain coplanar under $\varphi$ for every $Z^{\prime} \in \mathcal{I}_{Z}$. Eventually, this shows that all points of $Z$ remain coplanar under $\varphi$, and thus $Z$ is 0 -shear-resistant.

We now address the reverse implication: to this end, we first suppose that there exists $Z^{\prime} \in\left\{Z^{\text {out }}\right\} \cup \mathcal{I}_{Z}$ such that

$$
\min \left\{\# I_{1,+}\left(Z^{\prime}\right), \# I_{1,-}\left(Z^{\prime}\right)\right\}>1 \text { or } \min \left\{\# I_{2,+}\left(Z^{\prime}\right), \# I_{2,-}\left(Z^{\prime}\right)\right\}>1 .
$$

As $Z^{\prime}$ is simple, we can apply Proposition 6.1 to find a nontrivial angle-preserving mapping $\tilde{\varphi}$ of $Z^{\prime}$ such that the condition in Definition 5.1(i) is violated. It now suffices to check that $\tilde{\varphi}$ can be extended to an angle-preserving mapping on the whole of $Z$. To this end, denote the points of $Z^{\prime}$ by $\left\{\boldsymbol{p}^{1}, \ldots, \boldsymbol{p}^{m}\right\}$, and fix a connected component $\widehat{C}$ of $Z \backslash Z^{\prime}$. In view of our preliminary observation, we need to address two cases: (a) there is exactly one point $\boldsymbol{p}^{i} \in Z^{\prime}$ bonded to $\widehat{C}$ and (b) there are exactly two consecutive points $\boldsymbol{p}^{i}, \boldsymbol{p}^{i+1} \in Z^{\prime}$ bonded to $\widehat{C}$. In case (a), the three points $\boldsymbol{p}^{i-1}, \boldsymbol{p}^{i}, \boldsymbol{p}^{i+1}$ are contained in two lines and thus moved by the same isometry under $\tilde{\varphi}$, see the construction in (17). We denote this isometry by $R_{t}$ and set $\boldsymbol{\varphi}(\boldsymbol{x})=R_{t}(\boldsymbol{x})$ for all $\boldsymbol{x} \in \widehat{C}$. In case (b), the four points $\boldsymbol{p}^{i-1}, \boldsymbol{p}^{i}, \boldsymbol{p}^{i+1}, \boldsymbol{p}^{i+2}$ are contained in two lines and thus, as before, they are moved by a single isometry $R_{t}$ under $\tilde{\boldsymbol{\varphi}}$. We again set $\boldsymbol{\varphi}(\boldsymbol{x})=R_{t}(\boldsymbol{x})$ for all $\boldsymbol{x} \in \widehat{C}$. This construction leads to an angle-preserving mapping $\varphi$ defined on $Z$, which coincides with $\tilde{\varphi}$ on $Z^{\prime}$. This shows that $Z$ is not 0 -shear-resistant.

It remains to address the case

$$
Z \in \mathcal{F} \text { and } \min \left\{\# I_{1,+}\left(Z^{\prime}\right), \# I_{1,-}\left(Z^{\prime}\right)\right\}=\min \left\{\# I_{2,+}\left(Z^{\prime}\right), \# I_{2,-}\left(Z^{\prime}\right)\right\}=1
$$

for all $Z^{\prime} \in\left\{Z^{\text {out }}\right\} \cup \mathcal{I}_{Z}$. In this case, by Proposition 4.3 we find a nontrivial anglepreserving mapping $\varphi$ of $Z$ of the form indicated in Definition 4.2. Now, we need to check that the condition in Definition 5.1(i) is violated for at least one paraxial rectangle. In fact, by Proposition 6.1 we get that all points of $Z^{\text {out }}$ are coplanar under $\varphi$. On the other hand, by construction necessarily there exists a bond of $Z$ in the open region 
$f\left(Z^{\text {out }}\right)$ which does not remain coplanar to $Z^{\text {out }}$ under $\varphi$. Therefore, there exists a paraxial rectangle in $Z$ which does not remain coplanar under $\varphi$.

\section{ACKNOWLEDGEMENTS}

This work is partially supported by the FWF-DFG grant I 4354, the FWF grants F 65, I 5149, and P 32788, by the Vienna Science and Technology Fund (WWTF) through project MA14-009, and by the OeAD-WTZ project CZ 01/2021. This work was supported by the Deutsche Forschungsgemeinschaft (DFG, German Research Foundation) under Germany's Excellence Strategy EXC 2044 -390685587, Mathematics Münster: Dynamics-Geometry-Structure. The authors want to thank Jan Legerský for his helpful comments on a previous version of the manuscript.

\section{REFERENCES}

[1] N. L. Allinger. Molecular structure: understanding steric and electronic effects from molecular mechanics, Wiley, 2010.

[2] B. D. Anderson, C. Yu, B. Fidan, J. M. Hendrickx. Rigid graph control architectures for autonomous formations, IEEE Control Systems Magazine, 29 (2008), 48-63.

[3] L. Asimov, B. Roth. The rigidity of graphs, Trans. Amer. Math. Soc., 245 (1978), 279-289.

[4] X. Blanc, M. Lewin. The Crystallization Conjecture: a review. EMS Surv. in Math. Sci., 2 (2015), 255-306.

[5] D. W. Brenner. Empirical potential for hydrocarbons for use in stimulating the chemical vapor deposition of diamond films, Phys. Rev. B, 42 (1990), 9458-9471.

[6] I. Buckley, M. Egerstedt. Infinitesimally shape-similar motions using relative angle measurements, in Intelligent Robots and Systems (IROS), 2017 IEEE/RSJ International Conference. IEEE, 2017, pp. 1077-1082.

[7] L. Chen, M. Cao, C. Li. Bearing rigidity and formation stabilization for multiple rigid bodies in SE(3), Numer. Algebra Control Optim., 9 (2019), 257-267.

[8] W. E, D. Li. On the crystallization of 2D hexagonal lattices. Comm. Math. Phys., 286 (2009), 1099-1140.

[9] B. Farmer, S. Eseduglu, P. Smereka. Crystallization for a Brenner-like Potential. Comm. Math. Phys., 349 (2017), 1029-1061.

[10] L. C. Flatley, F. Theil. Face-centered cubic crystallization of atomistic configurations, Arch. Ration. Mech. Anal., 218 (2015), 363-416.

[11] G. Friesecke, F. Theil. Molecular geometry optimization, models, in Encyclopedia of Applied and Computational Mathematics, B. Engquist (ed.), Springer, Berlin, Heidelberg, 2015.

[12] G. Jing, G. Zhang, H. W. J. Lee, L. Wang. Weak rigidity theory and its application to formation stabilization. SIAM J. Control Optim., 56 (2018), 2248-2273.

[13] G. Jing, G. Zhang, H. W. J. Lee, L. Wang. Angle-based shape determination theory of planar graphs with application to formation stabilization. Automatica J. IFAC, 105 (2019), 117-129.

[14] D. Garamvölgyi, T. Jordán. Global rigidity of unit ball graphs, SIAM J. Discrete Math., 34 (2020), 212-229.

[15] B. Jackson, T. Jordan. Rigid components in molecular graphs. Algorithmica, 48 (2007), 399-412.

[16] B. Jackson, J. C. Owen. A characterisation of the generic rigidity of 2-dimensional point-line frameworks. J. Combin. Theory Ser. B, 119 (2016), 96-121.

[17] D. J. Jacobs, L. A. Kuhn, M. F. Thorpe. Flexible and rigid regions in proteins. In: Thorpe M.F., Duxbury P.M. (eds) Rigidity Theory and Applications. Fundamental Materials Research. Springer, Boston, 2002.

[18] N. Katoh, S. Tanigawa. A proof of the molecular conjecture. Discrete Comput. Geom., 45 (2011), $647-700$. 
[19] G. Laman. On graphs and the rigidity of plane skeletal structures, J. Engng. Math., 2 (1970), $331-340$

[20] E. G. Lewars. Computational Chemistry, 2nd edition, Springer, 2011.

[21] S. Liu. Origin and nature of bond rotation barriers: a unified view. J. Phys. Chem. A, 117 (2013), 962-965.

[22] L. Lovász. Graphs and geometry. American Mathematical Society Colloquium Publications, 65. American Mathematical Society, Providence, RI, 2019.

[23] E. Mainini, P. Piovano, U. Stefanelli. Finite crystallization in the square lattice, Nonlinearity, 27 (2014), 717-737.

[24] E. Mainini, U. Stefanelli. Crystallization in carbon nanostructures, Comm. Math. Phys., 328 (2014), $545-571$.

[25] K.-K. Oh, M.-C. Park, H.-S. Ahn. A survey of multi-agent formation control, Automatica, 53 (2015), 424-440.

[26] M.-C. Park, H.-K. Kim, H.-S. Ahn. Rigidity of distance-based formations with additional subtended-angle constraints, in Proceedings of the 17th International Conference on Control, Automation and Systems (ICCAS), 2017, pp. 111-116.

[27] B. Roth. Rigid and flexible frameworks, Amer. Math. Monthly, 88 (1981), 6-21.

[28] F. Saliola, W. Whiteley. Constraining plane configurations in CAD: circles, lines, and angles in the plane, SIAM J. Discrete Math., 18 (2004), 246-271.

[29] F. H. Stillinger, T. A. Weber. Computer simulation of local order in condensed phases of silicon, Phys. Rev. B, 8 (1985), 5262-5271.

[30] T.-S. Tay, W. Whiteley. Recent advances in the generic rigidity of structures. Structural Topology, 9 (1984), 31-38.

[31] J. Tersoff. New empirical approach for the structure and energy of covalent systems. Phys. Rev. B, 37 (1988), 6991-7000.

[32] W. Whiteley. Counting out to the flexibility of molecules, Phys. Biol., 2 (2005), S116.

(Laurent Bétermin) Institut Camille Jordan, Université Claude Bernard Lyon 1, 21 AVenue Claude Bernard, 69622 Villeurbanne Cedex, France

Email address: betermin@math.univ-lyon1.fr

$U R L:$ https://sites.google.com/site/homepagelaurentbetermin/

(Manuel Friedrich) Department of Mathematics, Friedrich-Alexander Universität ErlangenNürnberg. Cauerstr. 11, D-91058 Erlangen, Germany, \& Mathematics Münster, UniverSity OF MÜnster, Einsteinstr. 62, D-48149 Münster, Germany

Email address: manuel.friedrich@fau.de

$U R L$ : https://www. uni-muenster.de/AMM/en/Friedrich/

(Ulisse Stefanelli) Faculty of Mathematics, University of Vienna, Oskar-MorgensternPlatz 1, A-1090 Vienna, Austria, Vienna Research Platform on Accelerating Photoreaction Discovery, University of Vienna, Währingerstrasse 17, 1090 Wien, Austria, \& Istituto di Matematica Applicata e Tecnologie Informatiche E. Magenes, via Ferrata 1, I-27100 PAVIA, ITALY

Email address: ulisse.stefanelli@univie.ac.at

$U R L:$ http://www.mat.univie.ac.at/ stefanelli 